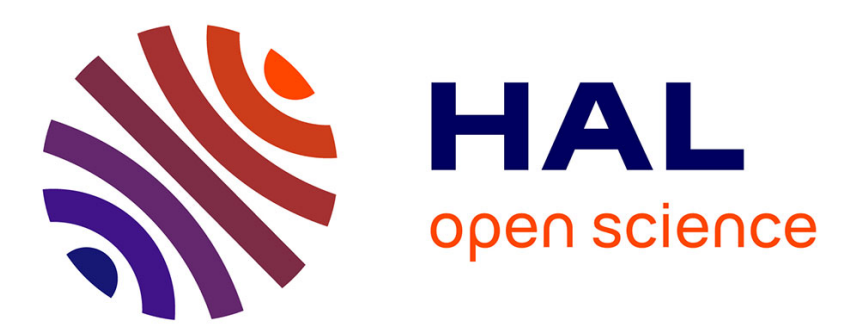

\title{
Modeling and optimization of hourglass-shaped aquaporins
}

Matthieu Bonnivard, Florian Omnès, Yannick Privat

\section{To cite this version:}

Matthieu Bonnivard, Florian Omnès, Yannick Privat. Modeling and optimization of hourglass-shaped aquaporins. Mathematical Models and Methods in Applied Sciences, 2018, 28 (8), pp.1529-1564. hal-01579886v3

\section{HAL Id: hal-01579886 https://hal.science/hal-01579886v3}

Submitted on 30 Sep 2020

HAL is a multi-disciplinary open access archive for the deposit and dissemination of scientific research documents, whether they are published or not. The documents may come from teaching and research institutions in France or abroad, or from public or private research centers.
L'archive ouverte pluridisciplinaire HAL, est destinée au dépôt et à la diffusion de documents scientifiques de niveau recherche, publiés ou non, émanant des établissements d'enseignement et de recherche français ou étrangers, des laboratoires publics ou privés. 


\title{
Modeling and optimization of hourglass-shaped aquaporins*
}

\author{
Matthieu Bonnivard ${ }^{\dagger} \quad$ Florian Omnès ${ }^{\ddagger} \quad$ Yannick Privat $^{\S}$
}

March 7, 2018

\begin{abstract}
This article is concerned with aquaporins (AQPs), that are proteins playing the role of water-selective channels also called nanopores, involved in many biological systems. From a technological point of view, it is relevant to design systems enjoying as good filtration properties. Inspired by [27], we investigate in a quite general framework shape optimization issues related to the improvement of hourglass-shaped aquaporins performances, in terms of energy dissipated by the fluid through the channel. After modeling this problem mathematically, we show that it is well-posed in some sense, and compute the so-called shape derivative of the cost functional in view of numerical simulations. Noting that our framework requires regularity properties of the free boundary, we introduce a dedicated numerical method, using in particular a proper shape gradient extension-regularization to adapt the mesh at each iteration, in an adequate way. Optimal shapes of aquaporins are then provided for relevant values of parameters, and we finally discuss the observed performances with respect to the existing results/literature.
\end{abstract}

Keywords: shape optimization, Navier-Stokes system, slip/flow rate boundary condition, extension-regularization procedure.

\section{Introduction and modeling of the problem}

\subsection{Motivations}

Aquaporins are proteins found in cell membrane in plants, bacteria and several organs of animals including humans. Along with membrane diffusion, aquaporins realize water filtration through the lipid bilayer, regulating the passage of water, ions and other solutes (see Fig. 1). The first aquaporin was fully identified in 1992, and was later named AQP1 [5]. At least ten other forms of AQPs have since been identified in mammals, in several body parts such as the kidney, eye and blood vessels [44]. Through experiments on humans and mice, it was shown that AQP1 is involved in many physiological processes, such as urin concentration, maintaining a proper intracranial pressure, and the production of aqueous fluid in the eye [12]. Aquaporin-based drugs could potentially offer treatments for diseases such as edematous states, cancer, obesity, epilepsy and glaucoma (see Verkman [46]).

\footnotetext{
*The authors were partially supported by the Project "Analysis and simulation of optimal shapes application to lifesciences" of the Paris City Hall.

${ }^{\dagger}$ Laboratoire Jacques-Louis Lions, Université Paris Diderot - Paris 7, 75013, Paris, France, (bonnivard@ljll.univ-paris-diderot.fr)

${ }^{\ddagger}$ Université Pierre et Marie Curie (Univ. Paris 6), CNRS UMR 7598, Laboratoire Jacques-Louis Lions, F-75005, Paris, France (florian.omnes@upmc.fr).

${ }^{\S}$ CNRS, Université Pierre et Marie Curie (Univ. Paris 6), UMR 7598, Laboratoire Jacques-Louis Lions, F-75005, Paris, France (yannick.privat@upmc.fr).
} 
Since the 1990s, the study of aquaporins has been an active field, both in describing their structure and understanding their function. For a full review of the topic, we refer to [12].

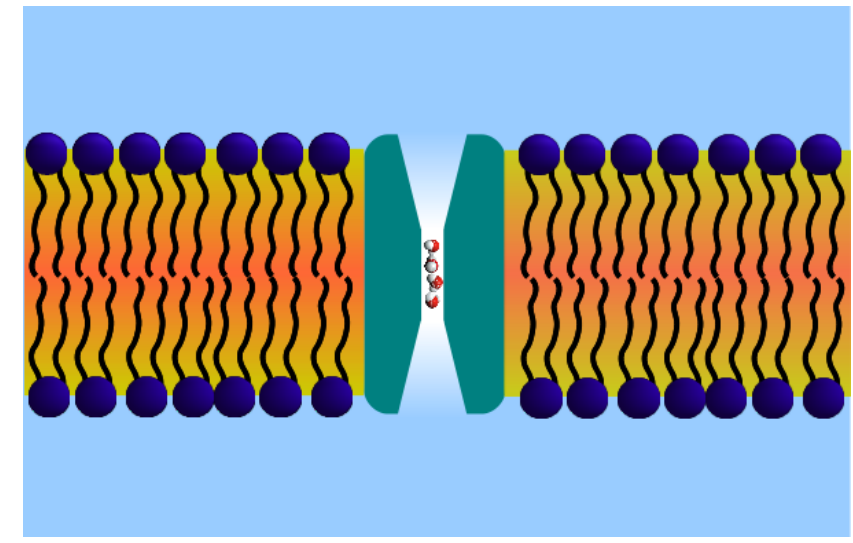

Figure 1: Scheme of an aquaporin. Water molecules are transported in a single file across the cell membrane, through an hourglass-shaped nanopore. Image courtesy of François Detcheverry (ILM, Lyon 1).

From a technological perspective, finding artificial systems enjoying high energy performance has applications in domains where membrane filtration is involved, from water desalination, to industrial food processes and wastewater treatment.

Water desalination is especially energy-intensive, and would likely benefit from improved energy efficiency. Two types of desalination plants are currently in operation: thermal-based and membrane-based. Only the latter type is discussed here. The principle of operation is as follows: pressurized soiled water is driven through a semi-permeable membrane which filters out the unwanted compounds. Reverse osmosis (RO) is widely used for desalination [24]. More generally, several technologies exist that allow to remove compounds within specific length-scale ranges: microfiltration $(0.1 \mu \mathrm{m}-1 \mu \mathrm{m})$, nanofiltration (NF) $(3 \mathrm{~nm}-30 \mathrm{~nm})$, and reverse osmosis $(0.1 \mathrm{~nm}-2 \mathrm{~nm})$ [36]. Similar but more recent than RO, NF features slightly larger pores. With a proven ability to remove pesticides, micro-pollutants, viruses and bacteria, NF could be used extensively to provide drinking water from groundwater or surface water [18].

Membrane-based filtration has also been adopted in the food industry [43]. Unlike conventional methods, membrane filtration allows cold filtration, which in-turn allows to preserve certain nutrients or aromas (ibid). Among many other applications, NF has been successfully used in the production of whey in the dairy industry [45].

In all these fields of applications of membrane filtering, an energy improvement is expected to provide significant cost reductions. This is especially true for desalination, where energy usually accounts for $25 \%-50 \%$ of total costs [34].

It is important to note however that for industrial applications, several factors must be taken into account:

- fouling, the process by which solid particles accumulate on membranes [24], lowering their efficiency. Fouled membranes must be replaced;

- membranes should be manufacturable at a small $(1 \mathrm{~nm}-10 \mathrm{~nm})$ scale;

- environmental impact [47, 21]. 
Notwithstanding, numerical simulation of membranes is expected to help imagining new efficient structures. Since the pioneering work of Hummer et al. [33], many simulations of fluid transport at a nanometric scale have been developed. These simulations can be classified into two categories:

- molecular dynamics (MD) models, that consider molecular interactions. See [48, 41] and [35, Chapter 16] for reference;

- methods based on continuum mechanics, that rely on the numerical resolution of the Navier-Stokes equations, using methods such as finite element methods (FEM) [27] or spectral elements methods [35, Chapter 14].

To the authors' best knowledge, MD have not yet been used to deal with shapeoptimization problems. However, testing on a wide range of parameters, MD and FEM have been shown to provide highly similar results for hydrodynamic resistance [27], even though the continuum hypothesis is usually not believed to hold at a $10 \mathrm{~nm}$ scale, the estimated diameter of an aquaporin channel. Previous experimental works also indicate that the Navier-Stokes equations hold when the channel diameter is more than ten times the size of a single fluid molecule [35, Chapter 10]. For water, this critical diameter size is roughly $3 \mathrm{~nm}$.

These considerations justify the use of FEM-based methods for simulating the transport of fluids by aquaporins.

Unlike MD simulations, FEM deal with continuous variables, making possible the use of the classical frameworks of fluid mechanics and shape-optimization. Note that optimizing molecular configurations seems challenging. Indeed, the computational cost associated to MD is usually high. Moreover, since only individual features of molecules or atoms are computed (speed, position), pressure and shear stress must be deduced in a second step. Yet, sensitivity analysis of energy functionals requires a good approximation of these quantities.

The main downside of our modeling choice is that it does not permit to deal with manufacturing constraints associated to the atomic length scale. Another limitation is that solid particles, which are known to influence the flow behavior when their volumic fraction becomes significant [35, Chapter 10], are omitted altogether.

In [26], Gravelle et al. investigate an hourglass model for the aquaporin. From physical considerations, they impose partial-slip boundary conditions for the fluid on the channel walls. Varying the angle of the inlet and outlet cones, they show numerically that there exists an optimal angle minimizing the energy dissipation by the fluid inside the water channel. The class of admissible shapes is extended in [3], where the shape of the inlet cone is optimized through three design parameters. The optimal design parameters are found by trying numerous combinations, without using an optimization algorithm, as, for instance, a gradient method. While this approach is admittedly computationally costly, it revealed that there are no local minima for the hydrodynamic resistance in the three dimensional design space.

More generally, FEM computations have widely been used in numerical shape optimization. References on the topic include [39]. Reference [42] provides an example of geometric shape optimization in biomedicine, where relevant criteria are optimized to improve long-term graft durability in the heart. Following this trend, [37] introduces the "Free form" approach in combination with reduced basis to reduce the number of design parameters and improve overall computational efficiency. Finally, a complete review is available in [38]. 
Using a similar model as in [26], we are interested in generalizing their work, by considering a wide family of admissible shapes, with the aim of analyzing more deeply the relationships between the aquaporin shape and its efficiency for permeating fluid. In this view, we will tackle the issue of minimizing the energy dissipated by the fluid through the channel constituting the structure, noting that this criterion is directly related to the performances of aquaporins. Concerning the modeling issues, we will assume that the fluid flux is known at the inlet, and that zero normal stress conditions (of Neumann type) are imposed at the outlet. It is notable that, in general, a flux condition does not allow to close a fluid model. Nevertheless, in our case, we will make a choice following the approach developed in [22] and derive a particular boundary condition implying the inlet flux condition, with the help of a dual variable.

Eventually, having in mind manufacturing issues, we want to avoid too complex designs. To that end, we will impose that the aquaporin shape enjoys a symmetry property and that it is connected. As mentioned before, this work intends to further enlarge the set of admissible shapes from [3]-[26]. Notice that the authors of [3]-[26] focus on hydrodynamic resistance whereas the present work is dedicated to energy dissipation. These two quantities are known to be closely related, but we preferred to deal with the energy dissipated by the fluid which is the "natural" energy associated to the system we consider, as it will be emphasized in the sequel.

Furthermore, we will use the same range of physical parameters as in [3]-[26], which will allow a careful comparison of the results. In order to take these analyses a stage further, we introduce a shape optimization algorithm based on shape derivative computations for the cost functional, giving access to a wider class of shapes. Let us stress that the expression of the shape derivative appears a bit unusual and requires an adapted algorithmic approach to infer an efficient numerical scheme, that will be introduced in the last section of this article.

The article is organized as follows. In Section 1.2, we introduce the fluid model for aquaporins and gather some tools for the analysis of the resulting system of PDEs. In Section 1.3, the shape optimization problem aiming at improving the performances of aquaporins by modifying its shape is introduced. We then analyze this problem in Section 2 , investigating existence issues and determining a workable expression of the cost function shape derivative. Finally, Section 3 is devoted to numerical issues and constitutes the core of this article. In particular, we improve the results in [26] by introducing an efficient algorithm of gradient type. The detailed steps of the method are precisely described. We then make the parameter choices in the model and the method precise, and comment on the obtained numerical results.

\subsection{Geometry and fluid model}

This section is devoted to modeling issues. To precise the framework of our study, we define the admissible geometries, as well as the fluid model, including our choices of boundary conditions. 


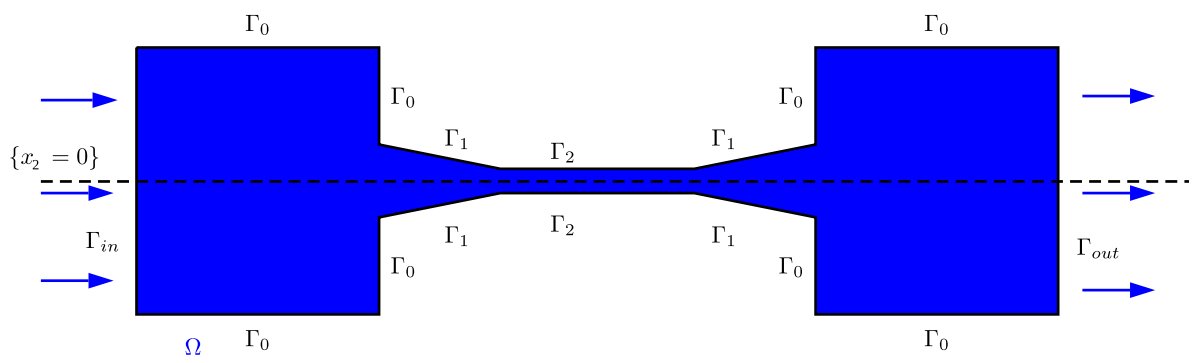

Figure 2: The domain $\Omega$ (two reservoirs connected by an hourglass shaped channel).

In what follows, in order to deal with realistic shapes, we will consider connected and bounded domains $\Omega$ in $\mathbb{R}^{2}$. Domain $\Omega$ describes the geometry of the aquaporin. An example of such $\Omega$ is depicted on Fig. 2. We assume the domain $\Omega$ to be filled with a viscous fluid of viscosity $\nu$, with $\nu>0$. The fluid domain $\Omega$ is made up of two reservoirs delimited by a lateral boundary $\Gamma_{0}$, and connected by a channel. The central part of the channel is tubular, its lateral boundary is denoted $\Gamma_{2}$; the inlet and outlet regions of the channel are conically shaped, with lateral boundary $\Gamma_{1}$. The upstream and downstream sections are labeled $\Gamma_{\text {in }}$ and $\Gamma_{\text {out }}$, respectively.

Notation. We denote by $\mathbf{n}$ the outward unit normal vector to $\partial \Omega$, and for every smooth vector field $\varphi$ defined on $\partial \Omega$, we define its tangential part by

$$
\varphi_{\tau}:=\varphi-(\varphi \cdot \mathbf{n}) \mathbf{n} .
$$

We define the strain tensor (symmetric part of the jacobian matrix $\nabla \mathbf{u}$ ) by

$$
\mathbf{D}(\mathbf{u})=\frac{1}{2}\left(\nabla \mathbf{u}+(\nabla \mathbf{u})^{T}\right),
$$

as well as the stress tensor

$$
\sigma(\mathbf{u}, p)=2 \nu \mathbf{D}(\mathbf{u})-p \mathrm{I}_{2},
$$

where $\mathbf{u}$ is the eulerian velocity of the fluid, $p$ is the pressure at every point $x \in \Omega$ and $\mathrm{I}_{2}$ is the identity matrix in $\mathbb{R}^{2 \times 2}$. We will denote by $\mathcal{H}^{1}$ the Hausdorff measure of dimension 1.

Fluid model and boundary conditions. The fluid motion is described by the Stokes equations

$$
\left\{\begin{array}{l}
-\operatorname{div}(\sigma(\mathbf{u}, p))=0 \quad \text { in } \Omega \\
\operatorname{div} \mathbf{u}=0 \text { in } \Omega
\end{array}\right.
$$

The momentum (1a) and mass-conservation (1b) equations are completed with boundary conditions. What follows is inspired by [6], where relevant boundary conditions on aquaporins are prescribed in order to obtain a closed physical fluid model, while prescribing the flux at the inlet of the considered structure.

- On the upstream section $\Gamma_{i n}$, we assume that only the flow rate of the fluid is given. This condition reads

$$
\int_{\Gamma_{\text {in }}} \mathbf{u} \cdot \mathbf{n} \mathrm{d} \mathcal{H}^{1}=-Q
$$

where the flow rate $Q$ is a nonzero real number and $\mathbf{n}$ is the outward normal vector. 
Recall that the average condition (2) is not sufficient to make the model well-posed and in particular to ensure the uniqueness of solutions. To overcome this difficulty, we follow the method developed in [22] to treat such "defective" boundary conditions involving averaged quantities instead of pointwise data on the boundary. Using this approach, condition (2) is interpreted as a (linear) contraint on the unknown $\mathbf{u}$ defined as a minimizer of an energy functional.

- In realistic applications, the conical regions of the channel are of nanometric size. At this scale, partial slip boundary conditions are considered relevant; they have been predicted theoretically and observed experimentally (see for instance [6]). Consequently, we set

$$
\mathbf{u} \cdot \mathbf{n}=0, \quad[\sigma(\mathbf{u}, p) \mathbf{n}]_{\tau}+\beta \mathbf{u}_{\tau}=0 \quad \text { on } \Gamma_{1},
$$

where $\beta>0$ is a constant friction parameter.

- Since the two reservoirs that are connected by the nanopore are typically of much larger size than the nanometric central channel, we impose the classical no-slip condition on their boundaries, that is,

$$
\mathbf{u}=0 \quad \text { on } \Gamma_{0} .
$$

- On the lateral boundary $\Gamma_{2}$ of the central (tubular) part of the channel, we neglect the frictional dissipation by assuming perfect slip boundary conditions

$$
\mathbf{u} \cdot \mathbf{n}=0, \quad[\sigma(\mathbf{u}, p) \mathbf{n}]_{\tau}=0 \quad \text { on } \Gamma_{2} .
$$

- Finally, on the downstream section $\Gamma_{\text {out }}$ of the domain, we consider free outflow conditions, modeled by Neumann boundary conditions

$$
\sigma(\mathbf{u}, p) \mathbf{n}=0 \quad \text { on } \Gamma_{\text {out }} .
$$

These boundary conditions are commonly used as passive conditions on artificial boundaries [32].

Let us introduce the functional space

$$
V(\Omega)=\left\{\varphi \in H^{1}\left(\Omega, \mathbb{R}^{2}\right), \quad \varphi_{\mid \Gamma_{0}}=0, \quad(\varphi \cdot \mathbf{n})_{\mid \Gamma_{1} \cup \Gamma_{2}}=0\right\} .
$$

Due to the no-slip boundary condition imposed on $\Gamma_{0}$, the Poincaré inequality holds in $V(\Omega)$ and reads

$$
\exists C>0, \quad \forall \varphi \in V(\Omega) \quad \int_{\Omega}|\varphi|^{2} \mathrm{~d} x \leq C \int_{\Omega}|\nabla \varphi|^{2} \mathrm{~d} x,
$$

where $|\cdot|$ stands either for the euclidian norm of a vector in $\mathbb{R}^{2}$, or a matrix in $\mathbb{R}^{2 \times 2}$, depending on the context. As a result, $V(\Omega)$ is a Hilbert space for the inner product $V(\Omega)^{2} \ni\left(\varphi_{1}, \varphi_{2}\right) \mapsto \int_{\Omega} \nabla \varphi_{1}: \nabla \varphi_{2} \mathrm{~d} x$. We denote by $\|\cdot\|_{V(\Omega)}$ the norm associated with this inner product.

In the sequel, we will also need to use Korn inequality, whose validity in $V(\Omega)$ is another consequence of the no-slip boundary condition imposed on $\Gamma_{0}$ and the Lipschitz regularity of the boundary $\partial \Omega$. This inequality reads

$$
\exists C_{K}>0, \quad \forall \varphi \in V(\Omega) \quad \int_{\Omega}|\nabla \varphi|^{2} \mathrm{~d} x \leq C_{K} \int_{\Omega}|\mathbf{D}(\varphi)|^{2} \mathrm{~d} x .
$$


Mixed formulation of the Stokes problem with imposed inner flow through $\Gamma_{i n}$.

For a given $Q \in \mathbb{R} \backslash\{0\}$, we consider the following problem: find $\left(\mathbf{u}_{\lambda}, p_{\lambda}\right) \in V(\Omega) \times L^{2}(\Omega)$ and $\lambda \in \mathbb{R}$ such that

$$
\left\{\begin{array}{l}
\forall \varphi \in V(\Omega) \quad 2 \nu \int_{\Omega} \mathbf{D}\left(\mathbf{u}_{\lambda}\right): \mathbf{D}(\varphi) \mathrm{d} x+\beta \int_{\Gamma_{1}} \mathbf{u}_{\lambda} \cdot \varphi \mathrm{d} \mathcal{H}^{1} \\
\quad-\int_{\Omega} p_{\lambda} \operatorname{div} \varphi \mathrm{d} x=\lambda \int_{\Gamma_{i n}} \varphi \cdot \mathbf{n} \mathrm{d} \mathcal{H}^{1} \\
\forall q \in L^{2}(\Omega) \quad \int_{\Omega} q \operatorname{div} \mathbf{u}_{\lambda} \mathrm{d} x=0 \\
\int_{\Gamma_{i n}} \mathbf{u}_{\lambda} \cdot \mathbf{n} \mathrm{d} \mathcal{H}^{1}=-Q
\end{array}\right.
$$

Remark 1.1. The parameter $\lambda$ appearing in (8a) can be regarded as the Lagrange multiplier associated to the constraint $\int_{\Gamma_{i n}} \mathbf{u}_{\lambda} \cdot \mathbf{n} \mathrm{d} \mathcal{H}^{1}=-Q$. Since both the constraint and the equations are linear, it will be made visible in the sequel that $-\lambda$ corresponds to the value of the normal constraint imposed on $\Gamma_{\text {in }}$ to obtain the desired flow rate.

In view of showing the well-posed character of this variational equation, we state an "inf-sup" type lemma adapted to the definition of the space $V(\Omega)$.

Lemma 1.2. The spaces $V(\Omega)$ and $L^{2}(\Omega)$ satisfy the inf-sup condition

$$
\inf _{q \in L^{2}(\Omega) \backslash\{0\}} \sup _{\varphi \in V(\Omega) \backslash\{0\}} \frac{\int_{\Omega} q \operatorname{div} \varphi \mathrm{d} x}{\|q\|_{L^{2}(\Omega)}\|\varphi\|_{V(\Omega)}}>0 .
$$

For the sake of clarity, the proof of this lemma is postponed to Section A.

The next proposition allows to interpret the solution $\mathbf{u}_{\lambda}$ of the Stokes system as a minimizer of an energy over a functional space.

Proposition 1.3. For every $Q \in \mathbb{R} \backslash\{0\}$, there exists a unique triple $\left(\mathbf{u}_{\lambda}, p_{\lambda}, \lambda\right) \in V(\Omega) \times$ $L^{2}(\Omega) \times \mathbb{R}$ satisfying $(8 \mathrm{a})-(8 \mathrm{~b})-(8 \mathrm{c})$.

Moreover, the function $\mathbf{u}_{\lambda}$ is the unique minimizer of the energy functional $\mathcal{E}_{\Omega}$ defined by

$$
\mathcal{E}_{\Omega}(\mathbf{w})=\nu \int_{\Omega}|\mathbf{D}(\mathbf{w})|^{2} \mathrm{~d} x+\frac{\beta}{2} \int_{\Gamma_{1}}|\mathbf{w}|^{2} \mathrm{~d} \mathcal{H}^{1}
$$

over the space

$$
V_{\text {div }}(\Omega)=V(\Omega) \cap\left\{\mathbf{w} \in H^{1}\left(\Omega, \mathbb{R}^{2}\right) \mid \operatorname{div} \mathbf{w}=0 \text { in } \Omega \text { and } \int_{\Gamma_{\text {in }}} \mathbf{w} \cdot \mathbf{n} \mathrm{d} \mathcal{H}^{1}=-Q\right\} .
$$

The proof of this proposition is postponed to Section B.

Remark 1.4. Notice that (8a)-(8b)-(8c) is the weak formulation of the partial differential equation

$$
\left\{\begin{array}{cl}
-\operatorname{div}\left(2 \nu \mathbf{D}\left(\mathbf{u}_{\lambda}\right)\right)+\nabla p_{\lambda}=0 & \text { in } \Omega, \\
\operatorname{div}\left(\mathbf{u}_{\lambda}\right)=0 & \text { in } \Omega, \\
\mathbf{u}_{\lambda}=0 & \text { on } \Gamma_{0} \\
\sigma\left(\mathbf{u}_{\lambda}, p_{\lambda}\right) \mathbf{n}+\lambda \mathbf{n}=0 & \text { on } \Gamma_{\text {in }} \\
\sigma\left(\mathbf{u}_{\lambda}, p_{\lambda}\right) \mathbf{n}=0 & \text { on } \Gamma_{\text {out }} \\
{\left[\sigma\left(\mathbf{u}_{\lambda}, p_{\lambda}\right) \mathbf{n}+\beta \mathbf{u}_{\lambda}\right]_{\tau}=0, \quad \mathbf{u}_{\lambda} \cdot \mathbf{n}=0} & \text { on } \Gamma_{1}, \\
{\left[\sigma\left(\mathbf{u}_{\lambda}, p_{\lambda}\right) \mathbf{n}\right]_{\tau}=0, \quad \mathbf{u}_{\lambda} \cdot \mathbf{n}=0} & \text { on } \Gamma_{2} .
\end{array}\right.
$$


According to the proof of Proposition 1.3, we claim that

$$
\lambda=-\frac{Q}{\int_{\Gamma_{i n}} \mathbf{u}_{1} \cdot \mathbf{n} \mathrm{d} \mathcal{H}^{1}},
$$

$\left(\mathbf{u}_{1}, p_{1}\right)$ being the solution of $(8 \mathrm{a})-(8 \mathrm{~b})$ with $\lambda=1$.

This can be obtained by combining the two following facts: first, fixing $\lambda \in \mathbb{R}$, the system (11) has a unique weak solution (this is a byproduct of Proposition 1.3). Second, the mapping $\mathbb{R} \ni \lambda \mapsto\left(\mathbf{u}_{\lambda}, p_{\lambda}\right)$, where $\left(\mathbf{u}_{\lambda}, p_{\lambda}\right)$ denotes the unique weak solution of system (11), is linear.

Finally, we end this section by investigating the consequence of the symmetry assumptions on the domain $\Omega$.

Mixed formulation of the Stokes problem with a symmetry condition. In this paragraph, we adapt our model to the case where $\Omega$ is symmetric with respect to the axis $\left\{x_{2}=0\right\}$, that will be addressed numerically in Sec. 3. To this aim, we introduce some extra notation. We denote by $\mathcal{H}$ the hyperplane $\mathcal{H}=\left\{x_{2}=0\right\}$, and by $\operatorname{Ref}_{\mathcal{H}}$ the reflexion through $\mathcal{H}$. We define $\mathcal{H}_{+}=\left\{x_{2}>0\right\}, \Omega_{+}=\Omega \cap \mathcal{H}_{+}$the upper part of the domain, and $\Gamma_{\text {sym }}=\Omega \cap \mathcal{H}$ its lower boundary.

If $\Omega$ is symmetric with respect to $\mathcal{H}$, then regular solutions of Stokes problem (8a)(8b)-(8c) enjoy nice symmetry properties, as stated in the following proposition.

Proposition 1.5. Assuming that the solution $(\mathbf{u}, p)$ to the Stokes system (8a)-(8b)-(8c) belongs to $H^{2}\left(\Omega, \mathbb{R}^{2}\right) \times H^{1}(\Omega)$,

$$
\mathbf{u}=\operatorname{Ref}_{\mathcal{H}}\left(\mathbf{u} \circ \operatorname{Ref}_{\mathcal{H}}\right) \quad \text { and } p=p \circ \operatorname{Ref}_{\mathcal{H}} \quad \text { a.e. in } \Omega,
$$

and as a consequence,

$$
[\sigma(\mathbf{u}, p) \mathbf{n}]_{\tau}=0 \quad \text { and } \quad \mathbf{u} \cdot \mathbf{n}=0 \text { on } \Gamma_{\text {sym }} .
$$

The proof of this proposition is postponed to Section C.

Hence, in the symmetric case, the flow is fully described by its restriction to the upper part $\Omega_{+}$of the domain. Besides, using the symmetry boundary condition on $\Gamma_{\text {sym }}(14)$ leads to modifying the weak formulation (8a)-(8b)-(8c) as follows: for $Q \in \mathbb{R} \backslash\{0\}$, find $\left(\mathbf{u}_{\lambda}, p_{\lambda}\right) \in \tilde{V}\left(\Omega_{+}\right) \times L^{2}\left(\Omega_{+}\right)$and $\lambda \in \mathbb{R}$ such that

$$
\left\{\begin{array}{l}
\forall \varphi \in \tilde{V}\left(\Omega_{+}\right), \quad 2 \nu \int_{\Omega_{+}} \mathbf{D}\left(\mathbf{u}_{\lambda}\right): \mathbf{D}(\varphi) \mathrm{d} x+\beta \int_{\Gamma_{1} \cap \mathcal{H}_{+}} \mathbf{u}_{\lambda} \cdot \varphi \mathrm{d} \mathcal{H}^{1} \\
\quad-\int_{\Omega_{+}} p_{\lambda} \operatorname{div} \varphi \mathrm{d} x=\lambda \int_{\Gamma_{i n} \cap \mathcal{H}_{+}} \varphi \cdot \mathbf{n} \mathrm{d} \mathcal{H}^{1} \\
\forall q \in L^{2}\left(\Omega_{+}\right) \quad \int_{\Omega_{+}} q \operatorname{div} \mathbf{u}_{\lambda} \mathrm{d} x=0 \\
\int_{\Gamma_{i n} \cap \mathcal{H}_{+}} \mathbf{u}_{\lambda} \cdot \mathbf{n} \mathrm{d} \mathcal{H}^{1}=-Q
\end{array}\right.
$$

where

$$
\tilde{V}\left(\Omega_{+}\right):=\left\{\varphi \in H^{1}\left(\Omega_{+}, \mathbb{R}^{2}\right), \quad \varphi_{\mid \Gamma_{0}}=0, \quad(\varphi \cdot \mathbf{n})_{\mid \Gamma_{1} \cup \Gamma_{2} \cup \Gamma_{\text {sym }}}=0\right\} .
$$

All the considerations of the previous paragraph still hold true in that case, justifying the well-posed character of this formulation. Moreover, Proposition 1.5 emphasizes that 
both formulations coincide when one assumes that $\Omega$ is symmetric w.r.t. the axis $\left\{x_{2}=0\right\}$, hence we can work with the simplified formulation (15a)-(15b)-(15c).

Dealing with symmetrical domains $\Omega$ will not only allow to integrate a kind of manufacturing constraint since it may appear difficult to design nonsymmetric shapes, but also to simplify the problem.

\subsection{The shape optimization problem}

From a physical point of view, it is reasonable to look for a shape minimizing the energy dissipated by the fluid inside the aquaporin. Indeed, physically, this criterion accounts for the viscous effects responsible for the irreversible conversion of mechanical energy into internal energy or heat.

The cost functional is defined by

$$
J(\Omega)=2 \nu \int_{\Omega}\left|\mathbf{D}\left(\mathbf{u}_{\Omega, \lambda}\right)\right|^{2} \mathrm{~d} x+\beta \int_{\Gamma_{1}}\left|\mathbf{u}_{\Omega, \lambda}\right|^{2} \mathrm{~d} \mathcal{H}^{1},
$$

where the triple $\left(\mathbf{u}_{\Omega, \lambda}, p_{\Omega, \lambda}, \lambda\right) \equiv\left(\mathbf{u}_{\lambda}, p_{\lambda}, \lambda\right) \in V(\Omega) \times L^{2}(\Omega) \times \mathbb{R}$ is defined in Proposition 1.3. Notice that $J(\Omega)$ also reads

$$
J(\Omega)=2 \min _{\mathbf{w} \in \tilde{V}_{\mathrm{div}}(\Omega)} \mathcal{E}_{\Omega}(\mathbf{w}),
$$

where $\tilde{V}_{\text {div }}(\Omega)$ and $\mathcal{E}_{\Omega}(\cdot)$ are defined in Proposition 1.3.

Since our main objective is to improve the results obtained in [26], we propose the following optimization strategy.

- First, we determine numerically the optimal angle of the inlet and outlet cones, minimizing the energy dissipation $J(\Omega)$ among all the domains $\Omega$ whose geometry is described by Figure 2. This step is a reproduction of the analysis performed in [26], and leads to obtaining a reference domain $\Omega^{*}$, characterized by the position of the conical part $\Gamma_{1}^{*}$ of its boundary (or equivalently, by the position of the terminal points of the lateral part $\Gamma_{0}^{*}$ ) .

- The improvement that we propose consists then in optimizing the shape of the conical regions of the domain, starting from the domain $\Omega^{*}$ that was determined in the previous step. In this process, the only part of the boundary that will be deformed is $\Gamma_{1}^{*}$; the rest of the boundary of $\Omega^{*}$ is fixed.

Let us give more details on the second step, that is, the shape optimisation of boundary $\Gamma^{1}$, starting from the optimal cone of boundary $\Gamma_{1}^{*}$. We define the class of admissible shapes by

$\mathcal{O}_{a d}=\left\{\Omega\right.$ open connected with a Lipschitz boundary, $\left.\Gamma_{\text {in }} \cup \Gamma_{\text {out }} \cup \Gamma_{0}^{*} \cup \Gamma_{2} \subset \partial \Omega\right\}$.

The resulting shape optimization problem reads

$$
\inf _{\Omega \in \mathcal{O}_{a d}} J(\Omega) .
$$

As previously stated, in the numerical simulations, we will restrict the admissible shapes to the ones that are symmetric with respect to the hyperplane $\mathcal{H}$. In that case, relying on Proposition 1.5, the cost functional can be expressed as

$$
J(\Omega)=2 J_{\text {sym }}\left(\Omega_{+}\right) \quad \text { where } \quad J_{\text {sym }}\left(\Omega_{+}\right)=2 \min _{\mathbf{w} \in \tilde{V}_{\text {div }}\left(\Omega_{+}\right)} \mathcal{E}_{\Omega_{+}}(\mathbf{w}),
$$

and

$$
\tilde{V}_{\operatorname{div}}\left(\Omega_{+}\right):=\left\{\varphi \in \tilde{V}\left(\Omega_{+}\right), \quad \operatorname{div} \varphi=0 \text { a.e on } \Omega^{+}\right\}
$$




\section{Analysis of the shape optimization problem}

This section is devoted first to the statement of an existence result for the shape optimization problem (18), and second, to the writing of the first order necessary optimality conditions for this problem.

\subsection{Existence issues}

It can be noted that the class $\mathcal{O}_{a d}$ defined by (17) is obviously not closed for usual domains topologies such as the Hausdorff complementary topology or the one associated to the strong $L^{1}$ convergence of characteristic functions. To avoid the emergence of irregular shapes, for which the PDE model described in Section 1.2 makes no sense, we choose to impose geometrical constraints on the free boundary $\Gamma_{1}$, the varying part of the geometry of the admissible sets.

First, one wants to deal with (at least) Lipschitz domains since the definition of the functional space $V(\Omega)$ involves the outward pointing normal vector, and since such regularity is required for using standard tools in the analysis of variational problems in Fluids Mechanics, such as Korn inequality. However, a minimizing sequence of Lipschitz domains may converge to a very irregular domain; we refer for instance to [1, 29] for examples of such ill-posed optimization problems. Notice that recent works (see [10, 9]) have highlighted that when considering shape optimization problems involving the solution of an elliptic PDE with Robin boundary conditions, minimizing sequences of domains may become very irregular and lead to the emergence of inner cracks.

A satisfying framework to deal with Robin boundary conditions in shape optimization has been introduced in [10,9]. It is based on a relaxation procedure, that consists in extending by 0 all test functions in the energy functional and embedding the free boundary problem into a larger class of functions, namely a subspace of special functions of bounded variation introduced originally by De Giorgi and Ambrosio. Unfortunately, adapting the approach of $[10,9]$ does not seem obvious. Indeed, this is due to

- the particular boundary conditions we consider, involving the normal and tangential parts of the vector field $\mathbf{u}$ and its derivative;

- the specificities of Fluids Mechanics equations, and in particular the divergence-free condition, which make it much more complicated to obtain a relaxed formulation of the PDE and the shape optimization problem (20). Notice also that the compactness theorems for SBV functions are not well adapted to dealing with symmetrized parts of gradients. In particular, it is not clear how to adapt the Korn inequality when considering domains with a boundary that is not Lipschitz regular.

A possible solution consists in restricting the class of admissible domains, by assuming some kind of uniform Lipschitz regularity. For that purpose, let us define the notion of $\varepsilon$-cone property, introduced in [13].

Definition 2.1. Let $y$ be a point of $\mathbb{R}^{2}, \xi$ a normalized vector and $\varepsilon>0$. We denote by $C(y, \xi, \varepsilon)$, the unpointed cone

$$
C(y, \xi, \varepsilon)=\left\{z \in \mathbb{R}^{2},\langle z-y, \xi\rangle \geq \cos \varepsilon\|z-y\| \text { and } 0<\|z-y\|<\varepsilon\right\} .
$$

We say that an open set $\Omega$ verifies the $\varepsilon$-cone property if

$$
\forall x \in \partial \Omega, \exists \xi_{x} \in \mathbb{S}^{1}, \forall y \in \bar{\Omega} \cap B(x, \varepsilon), C\left(y, \xi_{x}, \varepsilon\right) \subset \Omega .
$$


Another geometrical constraint, which is standard in shape optimization, is to assume that all admissible shapes are contained in a compact set $D$ to avoid the degeneracy of the free boundary. For this reason, let us introduce an external box $D$, defined as the convex hull of $\Omega$; in other words, $D$ is the rectangle of sides $\Gamma_{i n}, \Gamma_{\text {out }}$, and whose orthogonal sides contain the segments in $\Gamma_{0}$ that are parallel to the flow ${ }^{1}$ (see Fig. 3).

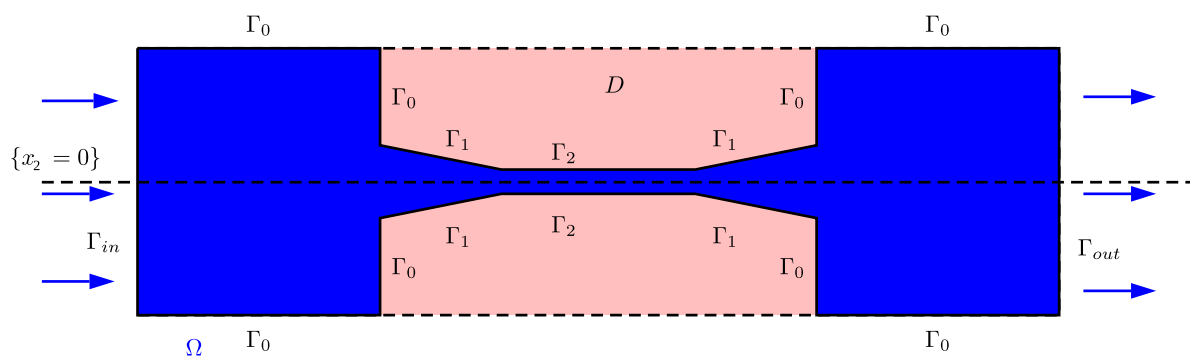

Figure 3: The external, rectangular box $D$ contains the domain $\Omega$ (in blue), completed with two lateral subregions (in pink).

Finally, the shape optimization problem that we investigate reads

$$
\inf \left\{J(\Omega), \Omega \in \mathcal{O}_{a d}, \Omega \subset D \text { and } \Omega \text { satisfies the } \varepsilon \text {-cone property }\right\},
$$

for some given parameter $\varepsilon>0$.

One has the following existence result.

Theorem 2.2. The shape optimization problem (20) has a solution.

The proof of this theorem is postponed to Section D.

Note that a similar existence result was obtained for a shape optimization problem arising in Fluid Mechanics with homogeneous Dirichlet conditions on the free boundary in $[30,31]$.

\subsection{Computation of the shape derivative of $J$}

We are interested in the differentiability of the solution $\mathbf{u}_{\lambda} \in V(\Omega)$ to system (8a)-(8b)(8c), with respect to deformations of the domain $\Omega$ preserving $\Gamma_{i n}, \Gamma_{\text {out }}, \Gamma_{0}$ and $\Gamma_{2}$, but acting on the shape of the "conical" boundary $\Gamma_{1}$. Let $\mathbf{V} \in W^{2, \infty}\left(\mathbb{R}^{2}, \mathbb{R}^{2}\right)$, with compact support, and such that $\mathbf{V}(x)=0$ if $x \in \partial \Omega \backslash \Gamma_{1}$. Let us stress that such $W^{2, \infty}$ regularity of the deformation field is specific to the treatment of a slip boundary condition in a stationary model of Newtonian flow. Indeed, in the case of Dirichlet boundary conditions, it is enough to consider Lipschitz deformations (see for instance [4]). In the present case, the recasting of the variational formulation of the problem in the reference domain requires to preserve the non penetration boundary condition on the the deformed boundary. This can be done by multiplying the velocity field by the Jacobian matrix of $\mathbf{V}$, to take into account the change of direction of the normal vector field. As a result, the energy associated with the system involves second derivatives of the displacement field, which explains the required smoothness on $\mathbf{V}$. We refer to Appendix $\mathrm{E}$ and the references therein for more details on this specific treatment of the partial slip boundary condition.

\footnotetext{
${ }^{1}$ Obviously, any other choice of compact set would be convenient from the point of view of existence theory. This particular choice is motivated by the biological/technological applications described in Section 1.1 .
} 
We introduce $T>0$ and a mapping

$$
t \in(-T, T) \mapsto \Phi_{t}=\left(\Phi_{t}^{1}, \Phi_{t}^{2}\right) \in W^{2, \infty}\left(\mathbb{R}^{2}, \mathbb{R}^{2}\right),
$$

of class $\mathcal{C}^{3}$, satisfying the properties

$$
\Phi_{0}=\mathrm{Id}, \quad{\frac{d \Phi_{t}}{d t}}_{\mid t=0}=\mathbf{V} .
$$

Moreover, we assume that $\Phi_{t}(x)=x$ for every $x \in \partial \Omega \backslash \Gamma_{1}$ and every $t \in(-T, T)$. A typical choice is given by $\Phi_{t}=\mathrm{Id}+t \mathbf{V}$.

We may choose $T$ small enough so that for $t \in(-T, T), \Phi_{t}$ is one to one and onto, and for every $x \in \mathbb{R}^{2}$, the mapping $t \in(-T, T) \mapsto \Phi_{t}^{-1}(x)$ is differentiable at $t=0$, with

$$
\frac{d}{d t}\left[\Phi_{t}^{-1}(x)\right]_{\mid t=0}=-\mathbf{V}(x) .
$$

For every $t \in(-T, T)$, we define $\Omega_{t}:=\Phi_{t}(\Omega)$ and denote by $\mathbf{u}_{\lambda, t} \in V\left(\Omega_{t}\right)$ the solution of system (8a)-(8b)-(8c) for $\Omega=\Omega_{t}$.

The proof of the next result is postponed to Section E for the sake of completeness.

Proposition 2.3. Let $\Omega \in \mathcal{O}_{a d}$. The mapping

$$
t \in(-T, T) \mapsto\left(\mathbf{u}_{\lambda, t} \circ \Phi_{t}, p_{\lambda, t} \circ \Phi_{t}\right) \in H^{1}(\Omega) \times L^{2}(\Omega)
$$

is differentiable at $t=0$.

In what follows, we will denote by $\langle d J(\Omega), \mathbf{V}\rangle$ the shape derivative of $J$ at $\Omega$ in the direction $\mathbf{V}$, in other words

$$
\langle d J(\Omega), \mathbf{V}\rangle=\lim _{t \searrow 0} \frac{J\left(\Omega_{t}\right)-J(\Omega)}{t} .
$$

From now on, we will assume at the same time more regularity on the domain $\Omega$ and on the vector field $\mathbf{V}$ in order to get a workable expression of the shape derivative. Hence, we will assume that $\partial \Omega$ is of class $C^{2}$ and $\mathbf{V} \in W^{3, \infty}\left(\mathbb{R}^{2}, \mathbb{R}^{2}\right)$. These properties ensure that the boundary of the domain $\Omega_{t}$ remains of class $C^{2}$, provided that $t$ is small enough (see e.g. [17]).

Theorem 2.4. Assume that $\partial \Omega$ is $C^{2}$. Let the triple $\left(\mathbf{u}_{\lambda}, p_{\lambda}, \lambda\right)$ be the unique solution to (8a)-(8b)-(8c). For every vector field $\mathbf{V} \in W^{3, \infty}$ having a compact support that does not intersect $\partial \Omega \backslash \Gamma_{1}$, there holds

$$
\langle d J(\Omega), \mathbf{V}\rangle=\int_{\Gamma_{1}} j_{1}(\mathbf{V} \cdot \mathbf{n})+j_{2} \cdot \nabla_{\Gamma}(\mathbf{V} \cdot \mathbf{n}) \mathrm{d} \mathcal{H}^{1}
$$

with

$$
\begin{aligned}
& j_{1}=2 \nu\left|\mathbf{D}\left(\mathbf{u}_{\lambda}\right)\right|^{2}+\beta\left(\partial_{n}\left(\left|\mathbf{u}_{\lambda}\right|^{2}\right)+H\left|\mathbf{u}_{\lambda}\right|^{2}\right)-2\left(\sigma\left(\mathbf{u}_{\lambda}, p_{\lambda}\right) \mathbf{n} \cdot \mathbf{n}\right) \partial_{n}\left(\mathbf{u}_{\lambda} \cdot \mathbf{n}\right) \\
& j_{2}=2\left(\sigma\left(\mathbf{u}_{\lambda}, p_{\lambda}\right) \mathbf{n} \cdot \mathbf{n}\right)\left[\mathbf{u}_{\lambda}\right]_{\tau}
\end{aligned}
$$

For the sake of clarity, the proof of Theorem 2.4 is postponed to section E.

So far, we have detailed the sensitivity analysis with general domains. However, a shape-derivative expression is necessary when considering a symmetric domain $\Omega$ as in the numerical simulations (Section 3). 
Corollary 2.5. Let $\Omega$ as in Theorem 2.4, with the additional hypothesis that $\Omega$ is symmetric through hyperplane $\mathcal{H}$ and $(\mathbf{u}, p)$ belongs to $H^{2}\left(\Omega, \mathbb{R}^{2}\right) \times H^{1}(\Omega)$, the shape derivative of $J_{\text {sym }}$ has the same expression as in Theorem 2.4, with a factor $\frac{1}{2}$, that is

$$
\left\langle d J_{\text {sym }}(\Omega), \mathbf{V}\right\rangle=\frac{1}{2} \int_{\Gamma_{1}} j_{1}(\mathbf{V} \cdot \mathbf{n})+j_{2} \cdot \nabla_{\Gamma}(\mathbf{V} \cdot \mathbf{n}) \mathrm{d} \mathcal{H}^{1}
$$

Proof. Notice that using Property 1.5 (and in particular (14)), problem (15a)-(15b)-(15c) is problem (8a)-(8b)-(8c) where $\Gamma_{2}$ has been replaced by $\Gamma_{2} \cup \Gamma_{\text {sym }}$. Thus proof of Corollary 2.5 is identical to that of Theorem 2.4 (appendix E), replacing $\Gamma_{2}$ with $\Gamma_{2} \cup \Gamma_{\text {sym }}$.

\section{Numerical methods and algorithms}

In this section, we will take advantage of the tools developed in Section 2 to infer an efficient algorithm for solving Problem (18). The numerical developments proposed in the sequel rely on the FreeFem++ [28] software, a free environment allowing to solve a wide variety of PDEs using the Finite Element method within a few command lines.

Let us first recall that in [26], the authors solved numerically a one-dimensional optimization problem, by assuming that each connected part of $\Gamma_{1}$ is a segment and making the inner angle between $\Gamma_{1}$ and $\Gamma_{2}$ vary. In a more recent article [3], shape optimization on hydrodynamic resistance is performed on a similar problem, assuming the cone wall is parametrized by a function depending on three parameters. A systematic (gradient-less) search is performed on the three parameters, and thus is computationally expensive. by

In an attempt to improve the results mentioned above, we will enrich their approach

- considering a wider class of admissible shapes for $\Gamma_{1}$,

- using numerical shape-optimization techniques based on the computation of the shape derivative.

We will solve the shape optimization problem (18), restricted the admissible shapes to symmetric ones, as stated in the last paragraph of Subsection 1.3. Notice that a close but simpler problem has been numerically investigated in [16]. In order to simplify notation, we present all the material on the full domain $\Omega$, but the calculations remain valid on the symmetric problem (see Corollary 2.5), just by replacing $\Omega$ by $\Omega_{+}, \Gamma_{1}$ by $\Gamma_{1} \cap \mathcal{H}_{+}, J$ by $J_{\text {sym }}$, etc.

Our approach can be decomposed into two main steps:

Step 1. Following [26], we recover the optimal inner angle between $\Gamma_{1}$ and $\Gamma_{2}$.

Step 2. Starting from the resulting straight cone with optimal angle, we find a local minimizer for the shape optimization problem (18), taking into account the symmetry constraint on the admissible shapes.

Let us stress that the proposed strategy is consistant with our main motivation, which is to reproduce the result presented in [26], and enhance the reduction of the cost functional by authorizing deformations of boundary $\Gamma_{1}$. Moreover, a global optimization of $J$ with respect to $\Omega$ would require to handle the junction points between $\Gamma_{0}$ and $\Gamma_{1}$, where a transition occurs between no-slip and partial slip boundary conditions. To our best knowledge, due to this change of boundary conditions, the treatment of deformations of the boundary $\Gamma_{0} \cap \Gamma_{1}$ would raise many difficulties, and would not be adapted to the tools developed within this article.

The two steps of the method are described in detail in the next two subsections. 


\subsection{Choice of parameters}

In order to perform numerical tests, reasonable physical parameters $\nu, \beta, Q$ as well as geometric dimensions for the aquaporin are required. In what follows, we will use that when the initial geometry $\Omega$ is fixed, the shape-optimization problem depends only on the ratio $\nu / \beta$.

To prove this, we introduce a normalized version of the energy, defined by

$$
\tilde{\mathcal{E}}_{\Omega}(\mathbf{w})=\frac{1}{\beta} \mathcal{E}_{\Omega}(\mathbf{w})=\frac{\nu}{\beta} \int_{\Omega}|D(\mathbf{w})|^{2} d x+\frac{1}{2} \int_{\Gamma_{1}}|\mathbf{w}|^{2} d \mathcal{H}^{2} .
$$

$\Omega$ being fixed, we see that the functional $\tilde{\mathcal{E}}_{\Omega}$ depends only the ratio $\nu / \beta$. Recall that $\mathbf{u}_{\lambda}$ is defined as the minimizer of this energy over the space $V_{d i v}(\Omega)$ :

$$
\tilde{\mathcal{E}}_{\Omega}\left(\mathbf{u}_{\lambda}\right)=\min \left\{\tilde{\mathcal{E}}_{\Omega}(\mathbf{w}), \mathbf{w} \in V_{\text {div }}(\Omega)\right\} .
$$

By this definition, it is clear that the minimum $\tilde{\mathcal{E}}_{\Omega}\left(\mathbf{u}_{\lambda}\right)$ depends only on $\nu / \beta$. We claim that the same holds for the minimizer $\mathbf{u}_{\lambda}$. This is a consequence of the construction of $\mathbf{u}_{\lambda}$, that is detailed in Appendix B. Indeed, using formulae (32)-(33) along with the definition of $\lambda$ from Eq. (12), we can write

$$
\lambda=-\frac{Q}{\int_{\Gamma_{i n}} \mathbf{u}_{1} \cdot \mathbf{n} d \mathcal{H}^{1}}=-\frac{2 \beta \tilde{\mathcal{E}}_{\Omega}\left(\mathbf{u}_{\lambda}\right)}{Q} .
$$

If $Q$ is fixed, the previous expression shows that $\lambda$ depends only on two parameters: $\beta$ (and the dependence is linear), and the ratio $\nu / \beta$. Finally, dividing Eq. (8a) by $\beta$ cancels out the dependence on $\beta$, and we obtain a normalized equation whose coefficients depend only on the same ratio $\nu / \beta$. Thus, its solution $\mathbf{u}_{\lambda}$ depends only on this ratio.

Using the same kind of argument, we see that the pair $\left(\mathbf{u}_{\lambda}, p_{\lambda}\right)$ depends linearly on $Q$, so the shape-optimization problem (20) is independent of $Q$. Subsequently, we choose $Q=1$.

Finally, following [27], we choose $L / a=20$, where $L$ is the length of the central tube, and $a$ is the central tube radius.

In what follows, we will concentrate on two relevant test cases to present our numerical results. All the parameters are chosen to be relevant for practical issues (see Table 1).

\begin{tabular}{|c|c|c|}
\hline & Test-case 1 & Test-case 2 \\
\hline$\beta$ & 1 & 1 \\
\hline$\nu$ & 20 & 100 \\
\hline$\tau_{0}$ & $5 \times 10^{-4}$ & $2 \times 10^{-4}$ \\
\hline$\varepsilon_{\text {stop }}$ & $10^{-1}$ & $10^{-1}$ \\
\hline$\theta^{*}$ & 0.265 & 0.311 \\
\hline
\end{tabular}

Table 1: Model and numerical parameters

\subsection{Finding the optimal angle (step 1 of the algorithm)}

We reproduce here the analysis in [26]. The goal of the first step is to find the optimal angle between $\Gamma_{1}$ and $\Gamma_{2}$. In other words, we solve the following optimization problem

$$
\inf \left\{J\left(\Omega_{\theta}\right), \theta \in\left[0, \theta_{\max }\right)\right\},
$$


$\Omega_{\theta}$ being the domain $\Omega$ with angle $\theta$ between $\Gamma_{1}$ and $\Gamma_{2}$. The upper part of this domain, along with a computational mesh is depicted in Fig. 4 for $\theta \in\{0.1,0.2,0.4\}$.

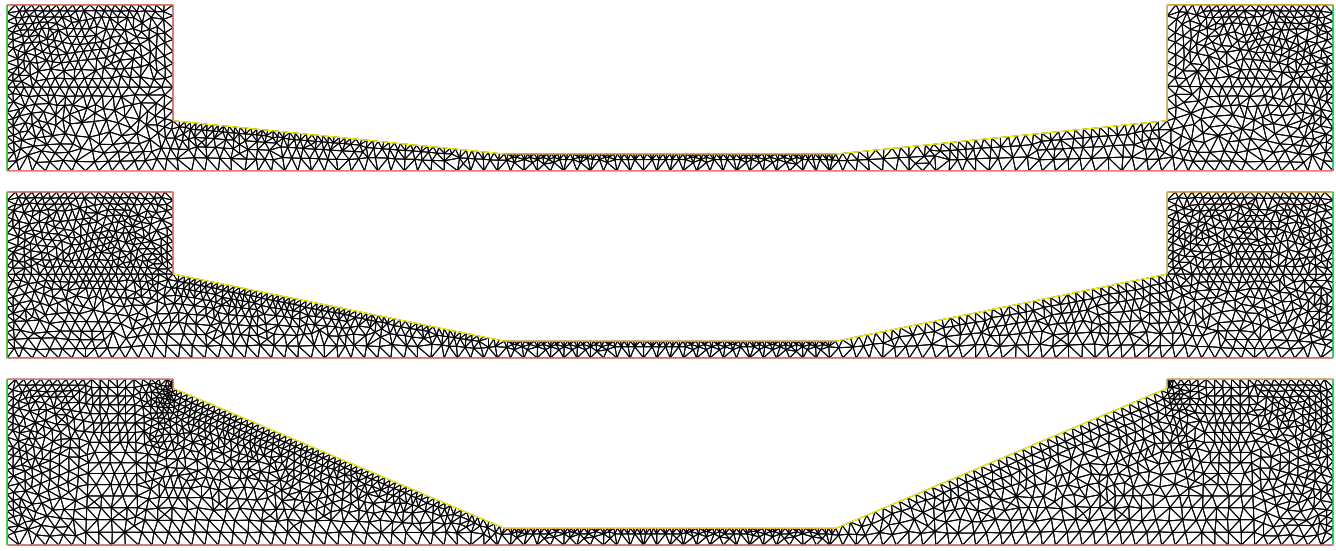

Figure 4: Computational domains for $\theta=0.1,0.2$ and 0.4 , from top to bottom.

For each value of $\theta$, a simplicial mesh on $\Omega_{\theta}$ is built, the Stokes equation (15a)-(15c) is solved using a standard finite elements method (FEM). The velocity and pressure are respectively approximated by $P_{2}$ and $P_{1}$ elements. From this solution, an approximated value of $J\left(\Omega_{\theta}\right)$ is computed.

In order to solve numerically problem (23), a simple dichotomy like procedure is used, making the angle between $\Gamma_{1}$ and $\Gamma_{2}$ vary. Fig. 5 shows the graph of mapping $\theta \mapsto J\left(\Omega_{\theta}\right)$ for cases 1 and 2. In this case, using for example the golden section line search [8] gives $\theta^{*}=0.265 \pm 0.001$, which is the value used in the second step. For test-case $2, \theta^{*}=$ $0.311 \pm 0.001$.
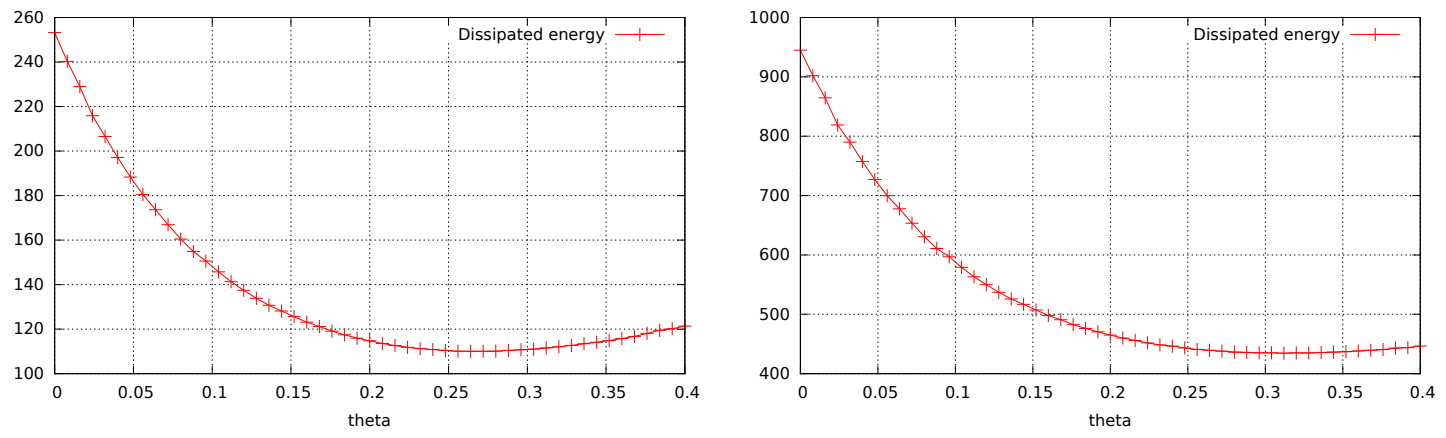

Figure 5: Criterion $J(\Omega)$ w.r.t. the angle parameter in Test-cases 1 and 2 (see Table 1).

\subsection{Optimizing the shape of $\Gamma_{1}$ (step 2 of the algorithm)}

In what follows, we will consider polygonal shapes $\Omega$, symmetric with respect to the hyperplane $\mathcal{H}=\left\{x_{2}=0\right\}$, and build a mesh $\mathcal{T}$ of their upper part $\Omega_{+}=\Omega \cap\left\{x_{2}>0\right\}$, composed of $K$ (closed) simplices $T_{1}, \ldots, T_{K}$ (i.e. triangles in $2 d$, tetrahedra in $3 d$ ), and $I$ vertices $\mathbf{x}_{1}, \ldots, \mathbf{x}_{I}$. This mesh is assumed to be a conforming simplicial covering-up of $\Omega_{+}[23]$.

Let us now provide the skeleton of the algorithm. 
- Initialization: Choose an initial admissible domain $\Omega$, symmetric with respect to $\mathcal{H}$. Define $\Omega^{0}:=\Omega_{+}$as the upper part of $\Omega$, and equip $\Omega^{0}$ with a mesh $\mathcal{T}^{0}$.

- For $n=0, \ldots$, until convergence:

1. Compute the solution $\left(\mathbf{u}_{\lambda}^{n}, p_{\lambda}^{n}\right) \in \tilde{V}\left(\Omega^{n}\right) \times L^{2}\left(\Omega^{n}\right)$ of Stokes equation (15a)-(15c), in $\Omega^{n}$, using the mesh $\mathcal{T}^{n}$.

2. Compute the shape derivative of $J\left(\Omega^{n}\right)$ (see Corollary 2.5) and infer a descent direction $\boldsymbol{\theta}^{n}$ for the optimization problem (Section 3.3).

3. Choose an appropriate gradient step $\tau^{n}$ and advect the shape $\Omega^{n}$ into the new shape $\Omega^{n+1}:=\left(\operatorname{Id}+\tau^{n} \boldsymbol{\theta}^{n}\right)\left(\Omega^{n}\right)$; a mesh $\mathcal{T}^{n+1}$ of $\Omega^{n+1}$ is obtained.

Convergence is reached whenever

$$
\frac{\left|J\left(\Omega^{n+1}\right)-J\left(\Omega^{n}\right)\right|}{\tau^{n}}<\varepsilon_{\text {stop }} .
$$

At each iteration, $\tau^{n}$ is initialized to a fixed value $\tau_{0}$ and divided by $q=1.5$ until

$$
J\left(\left(\operatorname{Id}+\tau^{n} \boldsymbol{\theta}^{n}\right)\left(\Omega^{n}\right)\right)<J\left(\Omega^{n}\right) .
$$

The mesh is finally advected point by point. Assuming the $i^{\text {th }}$ point of $\Omega^{n}$ has coordinates $\mathbf{x}_{i}^{n}$,

$$
\forall i \in\{1, \ldots, I\}, \quad \mathbf{x}_{i}^{n+1}=\mathbf{x}_{i}^{n}+\tau^{n} \boldsymbol{\theta}^{n}\left(\mathbf{x}_{i}^{n}\right) .
$$

This procedure will result in a valid mesh only if $\boldsymbol{\theta}^{n}$ is smooth enough and $\tau$ is small enough. If this is not the case, self-intersections can appear. We address this difficulty with the extension procedure of our algorithm.

The choice of $\varepsilon_{\text {stop }}$ has a minor impact on the final shape. Running the optimization algorithm on a fixed and large (typically 1000) number of steps, we noticed that

- Domains $\Omega^{n}$ converge to a fixed shape $\Omega^{*}$.

- After many iterations, the value of $J$ no longer decreases, no matter how small the gradient step $\tau_{0}$. This is because the shape gradient approximately zero. Note that in this case, one does not necessarily have $j_{1}=0$ and $j_{2}=0$ but rather $j_{1}-\operatorname{div}_{\Gamma}\left(j_{2}\right)=0$ using Equation (29). Again, we do not use this last quantity as a stopping criterion since it is difficult to compute accurately due to the presence of high-order derivatives of $\mathbf{u}$.

\section{Extension-regularization procedure}

In this section, we present the core of step 2. The method presented here relies on a $H^{1}$-regularization step [20, 11, 15], followed by a linear elasticity-based extension. To the authors knowledge, it is not standard, and allows to take into account $\int_{\Gamma_{1}} j_{2} \cdot \nabla_{\tau}(\boldsymbol{\theta} \cdot \mathbf{n}) \mathrm{d} \mathcal{H}^{1}$ without assuming additional regularity on the term $j_{2}$. 
Substep 1: regularization procedure. Let $U=H_{0}^{1}\left(\Gamma_{1}, \mathbb{R}^{2}\right)$ and let $\phi$ be the solution of the following PDE under variational form: find $\phi \in U$ such that for all $\psi \in U$,

$$
\int_{\Gamma_{1}} \nabla_{\Gamma} \phi \cdot \nabla_{\Gamma} \psi \mathrm{d} \mathcal{H}^{1}=-\int_{\Gamma_{1}} j_{1} \psi+j_{2} \cdot \nabla_{\Gamma} \psi \mathrm{d} \mathcal{H}^{1} \quad \text { for all } \psi \in U .
$$

The existence of $\phi$ is standard, by Lax-Milgram theorem. Taking now $\psi=\phi$ as test function in (25) yields

$$
\int_{\Gamma_{1}} j_{1} \phi+j_{2} \cdot \nabla_{\Gamma} \phi \mathrm{d} \mathcal{H}^{1}=-\int_{\Gamma_{1}}\left|\nabla_{\Gamma} \phi\right|^{2} \mathrm{~d} \mathcal{H}^{1} \leq 0 .
$$

Let us stress the importance of such a step, which provides a smooth function $\phi$ (in $\left.H_{0}^{1}\left(\Gamma_{1}, \mathbb{R}^{2}\right)\right)$ from the knowledge of $j_{1}$ and $j_{2}$ on $\Gamma_{1}$. Without this step, the algorithm produces increasingly distorted meshes, which are unsuitable for computation.

Substep 2: extension to the whole domain. We look for a vector field $\boldsymbol{\theta}$ satisfying at the same time

- $\boldsymbol{\theta} \cdot \mathbf{n}=\phi$ on $\Gamma_{1}$,

- and $\boldsymbol{\theta}$ is smooth inside $\Omega$.

For that purpose, we choose $\boldsymbol{\theta} \in H^{1}\left(\Omega, \mathbb{R}^{2}\right)$ as the unique solution of the linear elasticity problem

$$
\left\{\begin{array}{lrr}
-\operatorname{div}\left(\sigma_{e}(\boldsymbol{\theta})\right)=0 & \text { in } & \Omega \\
\boldsymbol{\theta}=0 & \text { on } & \Gamma_{\text {in }} \cup \Gamma_{\text {out }} \cup \Gamma_{0} \\
\boldsymbol{\theta} \cdot \mathbf{n}=\phi & \text { on } & \Gamma_{1} \\
{\left[\sigma_{e}(\boldsymbol{\theta}) \mathbf{n}\right]_{\tau}=0} & \text { on } & \Gamma_{1} \cup \Gamma_{\text {sym }} \\
\boldsymbol{\theta} \cdot \mathbf{n}=0 & \text { on } & \Gamma_{\text {sym }}
\end{array}\right.
$$

where $\sigma_{e}(\boldsymbol{\theta})$ stands for the elasticity tensor given by

$$
\sigma_{e}(\boldsymbol{\theta})=\mu_{e}\left(\nabla \boldsymbol{\theta}+(\nabla \boldsymbol{\theta})^{\top}\right)+\lambda_{e} \operatorname{div} \boldsymbol{\theta} .
$$

In practice, the parameters $\lambda_{e}$ and $\mu_{e}$ are fixed respectively to 1 and 0.5 .

This choice - which is widespread in meshing $[2,19,14]$ to help in keeping a mesh with fine quality - is motivated by the intuition that elastic displacements tend to induce little compression (i.e. local change in the volume).

It remains to show that, with the definitions above, the vector field $\boldsymbol{\theta}$ is a descent direction for $J$. According to Theorem 2.4, one has

$$
\begin{aligned}
\langle d J(\Omega), \boldsymbol{\theta}\rangle & =\int_{\Gamma_{1}} j_{1}(\boldsymbol{\theta} \cdot \mathbf{n}) \mathrm{d} \mathcal{H}^{1}+\int_{\Gamma_{1}} j_{2} \cdot \nabla_{\Gamma}(\boldsymbol{\theta} \cdot \mathbf{n}) \mathrm{d} \mathcal{H}^{1} \\
& =\int_{\Gamma_{1}} j_{1} \phi+j_{2} \cdot \nabla_{\Gamma} \phi \mathrm{d} \mathcal{H}^{1}=-\int_{\Gamma_{1}}\left|\nabla_{\Gamma} \phi\right|^{2} \mathrm{~d} \mathcal{H}^{1},
\end{aligned}
$$

since the expression of the shape derivative only depends on $\boldsymbol{\theta} \cdot \mathbf{n}$ on $\Gamma_{1}$. We then infer that taking $\mathbf{V}=\boldsymbol{\theta}$ as the solution of system (27) provides a descent direction for $J$.

As pointed out in [20, Section 3.4], choosing a good inner product on $\Gamma_{1}$ is crucial for implementation and algorithmic efficiency issues. Note that, in that case, taking for instance a $L^{2}$ inner product may produce irregular domains. The choice of a $H^{1}$ inner 
product on the manifold $\Gamma_{1}$ offers a better alternative both for stability and convergence speed.

The extension step produces a displacement field defined on $\Omega^{n}$. As mentioned in section $3.3, \boldsymbol{\theta}^{n}$ needs to be smooth to avoid invalid meshes. This is why the linear elasticity system is used (eq. (27)): the term $\lambda \operatorname{div}(\theta)$ is used to penalize local mesh compression, helping to avoid mesh self-intersections.

Let us conclude this paragraph with an important observation about the term $\int_{\Gamma_{1}} j_{2}$. $\nabla_{\tau}(\boldsymbol{\theta} \cdot \mathbf{n}) \mathrm{d} \mathcal{H}^{1}$ appearing in the expression of the shape derivative of $J$. For smooth data $\boldsymbol{\theta}, j_{2}$ and $\Gamma_{1}$, the following integration by parts formula [29, Theorem 5.4.13] provides

$$
\begin{aligned}
\int_{\Gamma_{1}} j_{2} \cdot \nabla_{\Gamma}(\theta \cdot \mathbf{n}) \mathrm{d} \mathcal{H}^{1} & =\int_{\Gamma}-(\theta \cdot \mathbf{n}) \operatorname{div}_{\Gamma}\left(j_{2}\right)+H(\theta \cdot \mathbf{n})\left(j_{2} \cdot \mathbf{n}\right) \mathrm{d} \mathcal{H}^{1} \\
& =\int_{\Gamma}-\operatorname{div}_{\Gamma}\left(j_{2}\right)(\theta \cdot \mathbf{n}) \mathrm{d} \mathcal{H}^{1} .
\end{aligned}
$$

The last line is obtained by noticing that $j_{2}$ is contained in the tangent plane a.e. on $\Gamma_{1}$. Using Theorem 2.4, the expression of the shape derivative of $J$ reduces to

$$
\langle d J, \boldsymbol{\theta}\rangle=\int_{\Gamma_{1}}\left(j_{1}-\operatorname{div}_{\Gamma}\left(j_{2}\right)\right)(\theta \cdot \mathbf{n}) \mathrm{d} \mathcal{H}^{1} .
$$

This remark should normally allow to use traditional regularization methods, as described in [20]. However, as seen in Theorem $2.4, j_{2}$ depends on first-order derivatives of $\mathbf{u}$ as well as the geometry of the domain. Formula (29) is therefore impractical for numerical purposes, since dealing with such a term would need to use high order finite elements and a very fine mesh, and would increase dramatically the cost of computation.

This is why the expression (29) is not directly used in the numerical algorithm we implemented. Notice that the term $j_{2}$ is in some sense regularized in the step (25).

\subsection{Numerical results}

From table 1 , recall that $\nu=20$ in test-case 1 whereas $\nu=100$ in test-case 2. This means that the relative effect of volumic dissipation compared to surface shear friction is expected to be more important in test-case 2 . 

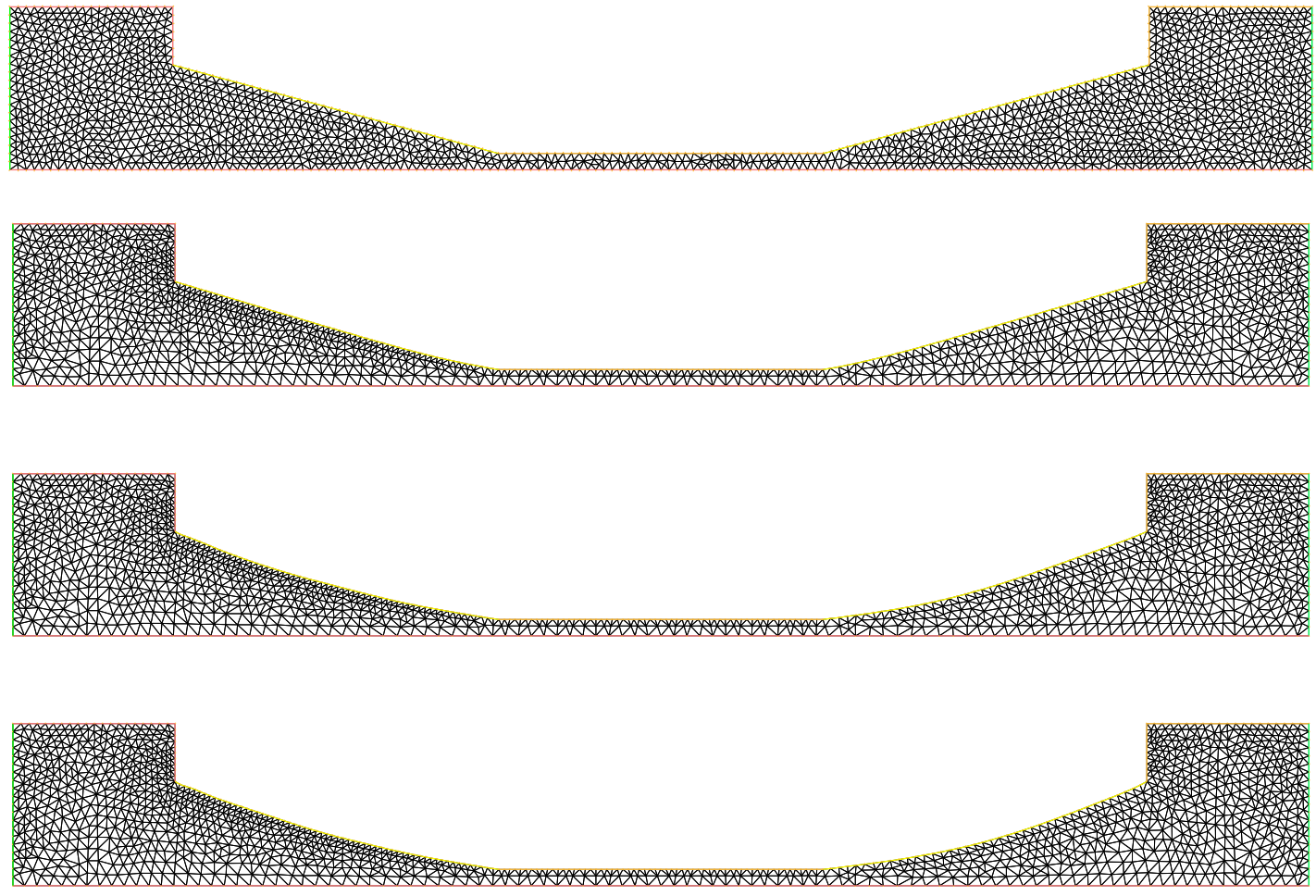

Figure 6: From top to bottom : mesh at iterations 0,45 and 85 for Test-case 1.
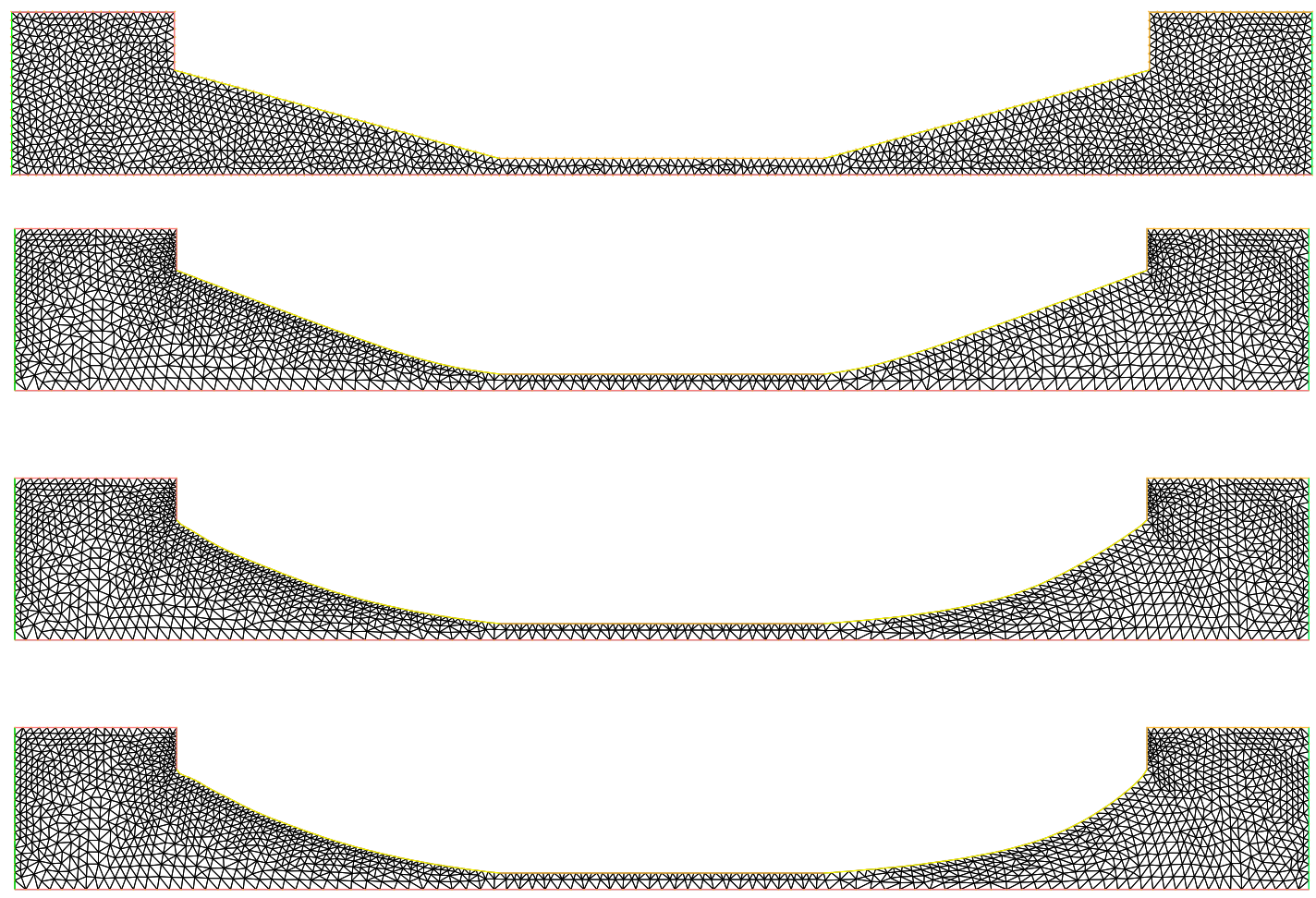

Figure 7: From top to bottom : mesh at iterations 0, 130 and 245 for Test-case 2. 
Comments. The step 2, described in Section 3.3, appears to be highly beneficial, leading to a $35 \%$ and $40 \%$ decrease of $J$ for test-cases 1 and 2 respectively. Several aquaporin profiles along the algorithm are displayed in Figures 6-7.

To take the analysis a step further, let us investigate which term in $J$ contributes most to $J$ between

- the viscous dissipation $J_{v}(\Omega):=2 \nu \int_{\Omega}\left|\mathbf{D}\left(\mathbf{u}_{\Omega, \lambda}\right)\right|^{2} \mathrm{~d} x$,

- the dissipation by friction $J_{f}(\Omega):=\beta \int_{\Gamma_{1}}\left|\mathbf{u}_{\Omega, \lambda}\right|^{2} \mathrm{~d} \mathcal{H}^{1}$.

Note that $J(\Omega)=J_{d}(\Omega)+J_{f}(\Omega)$.

In Figures 8-9, we observe that the reduction of the total dissipated energy achieved by step 2 of the algorithm, results from an important decrease of the viscous dissipation $J_{d}$, which appears to be the main contributor to the cost functional $J$. The frictional dissipation term $J_{f}$ accounts for less than 10 percent of the total dissipation, and is slightly increased during the process.
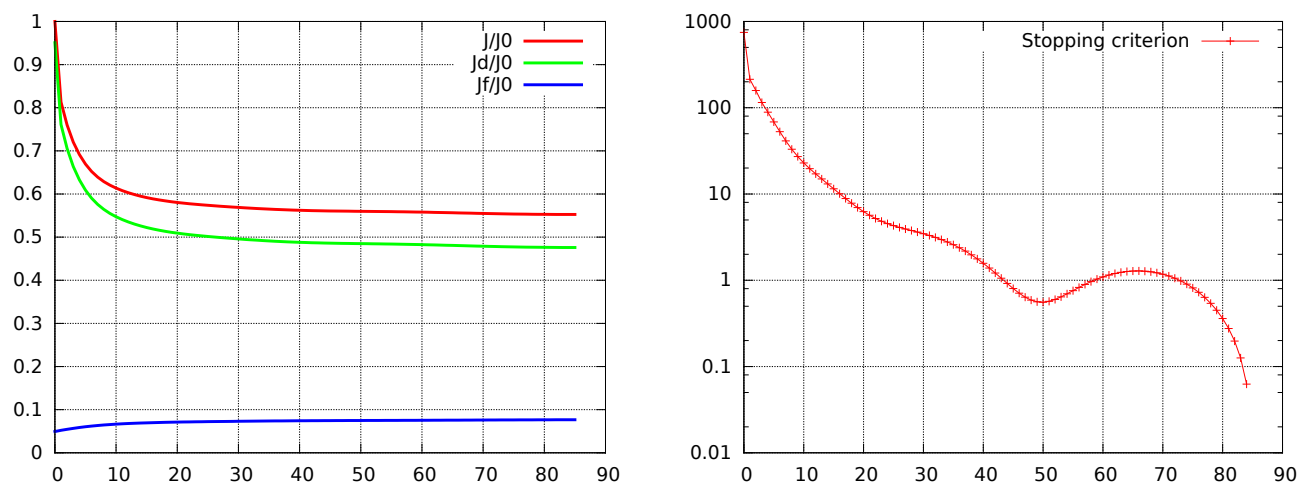

Figure 8: Test-case 1. Left: evolution of the cost functional $J$, and its subparts $J_{d}, J_{f}$ over iterations, relatively to the initial value $J_{0}$ of $J$. Right: evolution of the stopping criterion.
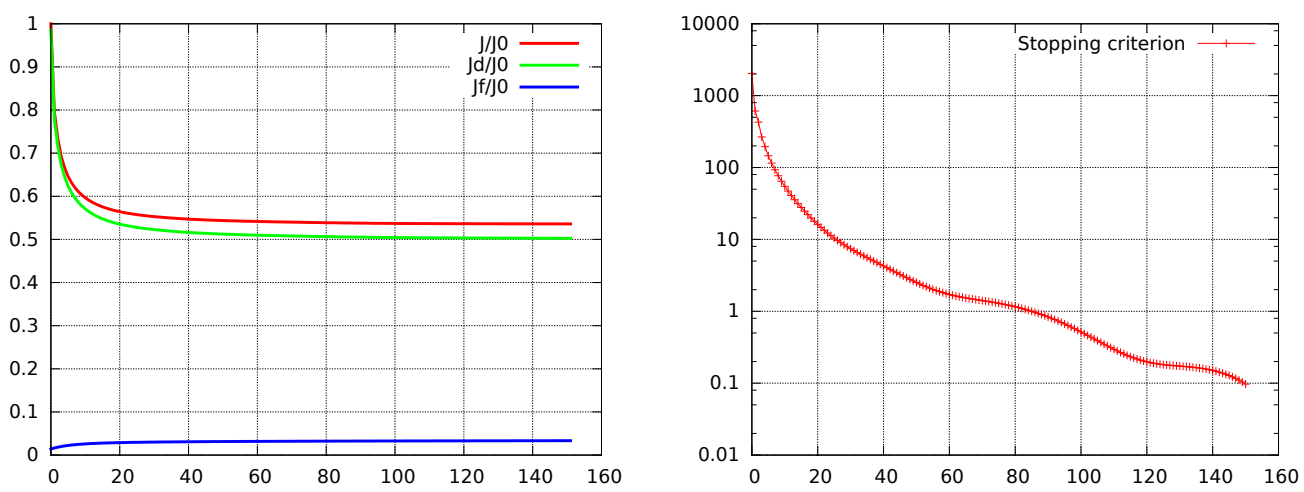

Figure 9: Test-case 2. Left: evolution of the cost functional $J$, and its subparts $J_{d}, J_{f}$ over iterations, relatively to the initial value $J_{0}$ of $J$. Right: evolution of the stopping criterion.

In what follows, we try to determine in which subdomain of $\Omega$ the criterion $J$ is decreased the most. For that purpose, one defines seven different regions as pictured on 
Fig. 10. On each of these regions $R_{i}$, we define

$$
J_{i}(\Omega)=2 \nu \int_{R_{i}}\left|\mathbf{D}\left(\mathbf{u}_{\Omega, \lambda}\right)\right|^{2} \mathrm{~d} x+\beta \int_{\overline{R_{i}} \cap \Gamma_{1}}\left|\mathbf{u}_{\Omega, \lambda}\right|^{2} \mathrm{~d} \mathcal{H}^{1}, \quad i \in\{1, \ldots, 7\} .
$$

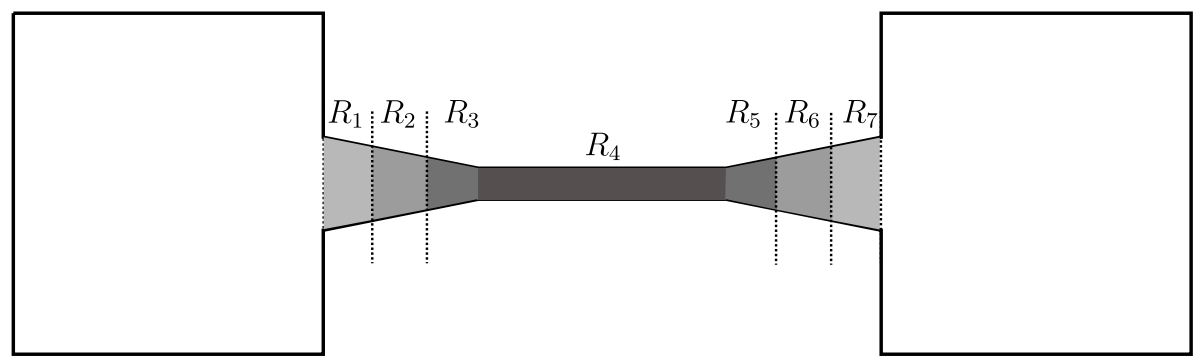

Figure 10: Outline of regions. Each region is defined as the intersection of $\Omega$ with an infinite vertical strip $\left\{a<x_{1}<b\right\}$. Regions 1, 2 and 3 (resp. 5, 6, 7) each take one third of the inlet (resp. outlet) cone width, region 4 is the whole central tube.
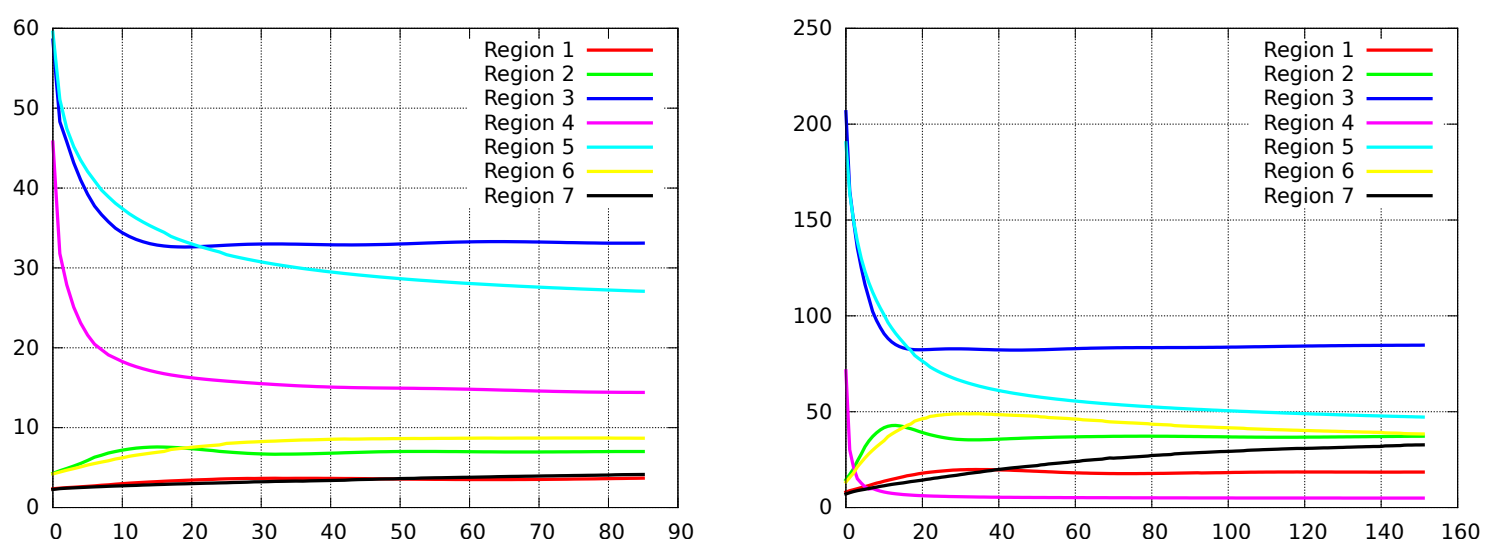

Figure 11: Evolution of each term $J_{i}$ during step 2 of the optimization process. Test-case 1 on the left, 2 on the right.

Note that $J$ is not the sum of all terms $J_{i}$, the reservoirs being excluded. From the previous observations, considering only the $J_{d}$ part of $J$ provides a reasonable qualitative estimate of $J$, but the $J_{f}$ part is also included for completeness. From Figure 11, it is visible that $J$ is mostly decreased in the central tube and in regions close to it. This is expected, since this is where most dissipation takes place. On the contrary, $J_{i}$ increases in other regions. This is not contradictory, since these regions only account for little dissipation. This can be seen as a tradeoff to minimize the most important effects, resulting in a decreasing $J$ in total. Finally, inlet and outlet dissipation (regions 1 and 7 ) looks almost unaffected. It may have been greatly decreased after step 1 (Section 3.2), leaving no space for further improvement.

\section{Further comments and conclusion}

On the initial value of $\theta$ and Step 1. As was highlighted in the description of the optimization process, the optimal shape is found by determining first the optimal angle $\theta^{*}$ 


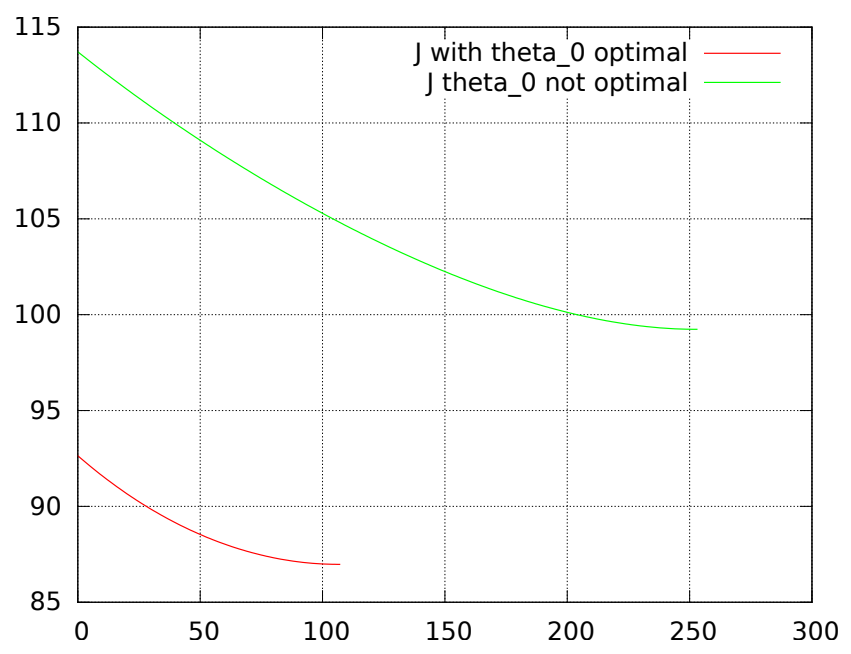

Figure 12: Plot of the objective functional during the iterations, starting with two different initial values for $\theta$. The red curve is obtained with $\theta^{*}$ extracted from Table 1 , the green curve is associated with $\theta=0.3$. The final value of $J$ is $21 \%$ lower when the initial angle $\theta^{*}$ is chosen.

between the aquaporin channel and the central part, and then by changing the profile of the channels (making the corresponding boundary vary without modifying its extremities). Figure 12 shows the importance of the first step. When choosing the best value for $\theta$ in the first step (namely, $\theta^{*}$ ), we observe that the criterion is smaller at initialization, but more importantly also at the end of the procedure.

Optimization and discretization of the problem In order to perform numerical experiments, it is necessary to choose a value for $h$, the typical mesh triangle size. If $h$ is too large, the evaluation of $J$ leads to numerical errors. On the other hand, the resolution of the Stokes equation becomes more expensive as $h$ becomes low. In order to have a good balance, we start from a coarse mesh and refine the iterates during the algorithm. This strategy is widespread in the literature, and fully detailed in [40].

Conclusion. We tried to enrich the approach presented in [27], in other words to improve the channel shapes that have been obtained and get a significant decrease of the objective function. Although we cannot compare precisely our results to those of [27], since the provided parameters are not sufficient to fully reproduce their simulations, Figure 5 reveals a similar (convex) profile for the function $\theta \mapsto J\left(\Omega_{\theta}\right)$, where $\Omega_{\theta}$ is the domain with straight channels making an angle $\theta$ with the central part. As shown in Figures 8 and 9, our strategy led to decreasing the objective function by at least $40 \%$, by comparison with the domain with straight channels and optimal angle $\theta^{*}$.

\section{A Proof of Lemma 1.2}

Before showing Lemma 1.2, we state a useful preliminary result.

Lemma A.1. There exists a constant $C>0$, depending only on $\Omega$, such that for every $q \in L^{2}(\Omega)$, there exists $\mathbf{v} \in V(\Omega)$ satisfying

$$
\operatorname{div} \mathbf{v}=q \quad \text { in } \Omega \quad \text { and } \quad\|\mathbf{v}\|_{V(\Omega)} \leq C\|q\|_{L^{2}(\Omega)} .
$$


Proof. Let $q \in L^{2}(\Omega)$, and consider $\alpha_{0} \in \mathcal{C}^{\infty}\left(\mathbb{R}^{2}\right)$, non identically null, with compact support, and such that $\left(\operatorname{spt} \alpha_{0}\right) \cap\left(\partial \Omega \backslash \Gamma_{i n}\right)=\emptyset$ and $\int_{\Gamma_{i n}} \alpha_{0} \cdot \mathbf{n} \mathrm{d} \mathcal{H}^{1}>0$. For every $x \in \Omega$, we define

$$
\alpha(x):=-\frac{\int_{\Omega} q(x) \mathrm{d} x}{\int_{\Gamma_{\text {in }}} \alpha_{0} \cdot \mathbf{n} \mathrm{d} \mathcal{H}^{1}} \alpha_{0}(x) .
$$

By construction, $\int_{\Gamma_{i n}} \alpha \cdot \mathbf{n} \mathrm{d} \mathcal{H}^{1}=-\int_{\Omega} q(x) \mathrm{d} x$ and by Hölder inequality and the boundedness of $\alpha_{0}$ and its derivatives, there exists a constant $C>0$ such that $\|\alpha\|_{H^{1}\left(\Omega, \mathbb{R}^{2}\right)} \leq$ $C\|q\|_{L^{2}\left(\Omega, \mathbb{R}^{2}\right)}$. Moreover, $q-\operatorname{div} \alpha \in L^{2}(\Omega)$, and using Stokes formula and the properties of the support of $\alpha$,

$$
\int_{\Omega}(q-\operatorname{div} \alpha) \mathrm{d} x=\int_{\Omega} q \mathrm{~d} x-\int_{\partial \Omega} \alpha \cdot \mathbf{n} \mathrm{d} \mathcal{H}^{1}=\int_{\Omega} q \mathrm{~d} x+\int_{\Gamma_{i n}} \alpha \cdot \mathbf{n} \mathrm{d} \mathcal{H}^{1}=0 .
$$

Thus, since $\Omega$ is a Lipschitz domain, there exists a constant $C>0$ depending only on $\Omega$, and a vector field $\mathbf{v}_{0} \in H_{0}^{1}\left(\Omega, \mathbb{R}^{2}\right)$ such that

$$
\operatorname{div} \mathbf{v}_{0}=q-\operatorname{div} \alpha \quad \text { and } \quad\left\|\mathbf{v}_{0}\right\|_{H_{0}^{1}(\Omega)} \leq C\|q-\operatorname{div} \alpha\|_{L^{2}(\Omega)}
$$

(see, for instance, [25] Corollary 2.4). Hence, $\left\|\mathbf{v}_{0}\right\|_{H^{1}\left(\Omega, \mathbb{R}^{2}\right)} \leq C\|q\|_{L^{2}\left(\Omega, \mathbb{R}^{2}\right)}$. Since $\alpha \in$ $V(\Omega)$, the function $\mathbf{v}$ defined by $\mathbf{v}=\mathbf{v}_{0}+\alpha$ belongs to $V(\Omega)$ and satisfies (31).

Let $q \in L^{2}(\Omega) \backslash\{0\}$ and $\mathbf{v} \in V(\Omega)$ such that (31) holds. Then

$$
\sup _{\varphi \in V(\Omega) \backslash\{0\}} \frac{\int_{\Omega} q \operatorname{div} \varphi}{\|q\|_{L^{2}(\Omega)}\|\varphi\|_{V(\Omega)}} \geq \frac{\int_{\Omega} q \operatorname{div} \mathbf{v}}{\|q\|_{L^{2}(\Omega)}\|\mathbf{v}\|_{V(\Omega)}}=\frac{\|q\|_{L^{2}(\Omega)}}{\|\mathbf{v}\|_{V(\Omega)}} \geq C^{-1}>0,
$$

where $C$ is defined in Lemma A.1. Taking the infimum over $q \in L^{2}(\Omega) \backslash\{0\}$ yields the desired result.

\section{B Proof of Proposition 1.3}

This proof is an adaptation of the proof of [22, Proposition 2], to the case of a mix of no-slip, partial slip and perfect slip conditions on different parts of the boundary of the domain. For the sake of completeness, we recall it briefly.

Existence of a solution. Let $\left(\mathbf{u}_{1}, p_{1}\right) \in V(\Omega) \times L^{2}(\Omega)$ be the solution to the following (unconstrained) mixed formulation:

$$
\begin{aligned}
& \forall \varphi \in V(\Omega) \quad 2 \nu \int_{\Omega} \mathbf{D}\left(\mathbf{u}_{1}\right): \mathbf{D}(\varphi) \mathrm{d} x+\beta \int_{\Gamma_{1}} \mathbf{u}_{1} \cdot \varphi \mathrm{d} \mathcal{H}^{1} \\
&-\int_{\Omega} p_{1} \operatorname{div} \varphi \mathrm{d} x=\int_{\Gamma_{i n}} \varphi \cdot \mathbf{n} \mathrm{d} \mathcal{H}^{1} \\
& \forall q \in L^{2}(\Omega) \quad \int_{\Omega} q \operatorname{div} \mathbf{u}_{1} \mathrm{~d} x=0
\end{aligned}
$$

By continuity of the trace operator $H^{1}\left(\Omega, \mathbb{R}^{2}\right) \rightarrow L^{2}\left(\Gamma_{i n}\right)$ and Hölder inequality, the linear operator $L: \varphi \in V(\Omega) \mapsto \int_{\Gamma_{\text {in }}} \varphi \cdot \mathbf{n} \mathrm{d} \mathcal{H}^{1}$ is bounded. Hence, the existence and uniqueness of such $\left(\mathbf{u}_{1}, p_{1}\right) \in V(\Omega) \times L^{2}(\Omega)$ result from Korn inequality (7) and the inf-sup condition (9) (see [25], Lemma 4.1). Now, we set

$$
\lambda=-\frac{Q}{\int_{\Gamma_{i n}} \mathbf{u}_{1} \cdot \mathbf{n} \mathrm{d} \mathcal{H}^{1}} .
$$


By linearity of equations (32)-(33), defining $\left(\mathbf{u}_{\lambda}, p_{\lambda}\right)=\lambda\left(\mathbf{u}_{1}, p_{1}\right)$, we obtain a solution to system (8a)-(8c).

Uniqueness of the solution. Let $(\mathbf{y}, r, \lambda),(\mathbf{z}, s, \mu) \in V(\Omega) \times L^{2}(\Omega) \times \mathbb{R}$ be two solutions of equations $(8 \mathrm{a})-(8 \mathrm{c})$. By linearity, we deduce from $(8 \mathrm{a})$ the relation

$$
\begin{aligned}
\forall \varphi \in & V(\Omega) \quad 2 \nu \int_{\Omega}(\mathbf{D}(\mathbf{y}-\mathbf{z})): \mathbf{D}(\varphi) \mathrm{d} x+\beta \int_{\Gamma_{1}}(\mathbf{y}-\mathbf{z}) \cdot \varphi \mathrm{d} \mathcal{H}^{1} \\
& -\int_{\Omega}(r-s) \operatorname{div} \varphi \mathrm{d} x-(\lambda-\mu) \int_{\Gamma_{i n}} \varphi \cdot \mathbf{n} \mathrm{d} \mathcal{H}^{1}=0 .
\end{aligned}
$$

Testing with $\varphi=\mathbf{y}-\mathbf{z}$ and using the relation $\int_{\Gamma_{i n}}(\mathbf{y}-\mathbf{z}) \cdot \mathbf{n} \mathrm{d} \mathcal{H}^{1}=0$, we obtain

$$
2 \nu \int_{\Omega}|\mathbf{D}(\mathbf{y}-\mathbf{z})|^{2} \mathrm{~d} x+\beta \int_{\Gamma_{1}}|\mathbf{y}-\mathbf{z}|^{2} \mathrm{~d} \mathcal{H}^{1}=0 .
$$

By Poincaré and Korn inequalities, this yields $\mathbf{y}=\mathbf{z}$ a.e. in $\Omega$. Now, testing with $\varphi=\mathbf{y}$ in (35) and using the constraint $\int_{\Gamma_{i n}} \mathbf{y} \cdot \mathbf{n} \mathrm{d} \mathcal{H}^{1}=Q$, we deduce $(\lambda-\mu) Q=0$, and so $\lambda=\mu$. Applying Lemma A.1, there exists $\mathbf{w} \in V(\Omega)$ such that $\operatorname{div} \mathbf{w}=r-s$ a.e. in $\Omega$. Thus, the relation $\int_{\Omega}(r-s) \operatorname{div} \mathbf{w} \mathrm{d} x=0$ yields $\|r-s\|_{L^{2}(\Omega)}=0$, which concludes the first part of the proof.

It remains to show that the function $\mathbf{u}_{\lambda}$ minimizes the energy functional $\mathcal{E}_{\Omega}$ over $V_{\text {div }}(\Omega)$. To that end, let us consider any $\mathbf{v} \in V_{\operatorname{div}}(\Omega)$ and write $\mathbf{v}=\mathbf{u}_{\lambda}+\mathbf{h}$. Then, $\mathbf{h} \in V(\Omega)$ is divergence free and satisfies $\int_{\Gamma_{i n}} \mathbf{h} \cdot \mathbf{n} \mathrm{d} \mathcal{H}^{1}=0$. Using that $\mathbf{u}_{\lambda}$ satisfies (8a), one computes

$$
\begin{aligned}
\mathcal{E}_{\Omega}(\mathbf{v})-\mathcal{E}_{\Omega}\left(\mathbf{u}_{\lambda}\right) & =\nu \int_{\Omega}|\mathbf{D}(\mathbf{h})|^{2} \mathrm{~d} x+\frac{\beta}{2} \int_{\Gamma_{1}}|\mathbf{h}|^{2} \mathrm{~d} \mathcal{H}^{1}-\lambda \int_{\Gamma_{i n}} \mathbf{h} \cdot \mathbf{n} \mathrm{d} \mathcal{H}^{1} \\
& =\nu \int_{\Omega}|\mathbf{D}(\mathbf{h})|^{2} \mathrm{~d} x+\frac{\beta}{2} \int_{\Gamma_{1}}|\mathbf{h}|^{2} \mathrm{~d} \mathcal{H}^{1} \geq 0 .
\end{aligned}
$$

The expected conclusion follows.

\section{Proof of Proposition 1.5}

Let us use the following notation: for a vectorial function $\mathbf{y}$, we define $\widetilde{\mathbf{y}}=\operatorname{Ref}_{\mathcal{H}}\left(\mathbf{y} \circ \operatorname{Ref}_{\mathcal{H}}\right)$ and for a scalar function $s$, we define $\widetilde{s}=s \circ \operatorname{Ref}_{\mathcal{H}}$. For all $(\varphi, q) \in V(\Omega) \times L^{2}(\Omega)$, one has

$$
\begin{aligned}
& 2 \nu \int_{\Omega} \mathbf{D}(\widetilde{\mathbf{u}}): \mathbf{D}(\varphi) \mathrm{d} x+\beta \int_{\Gamma_{1}} \widetilde{\mathbf{u}} \cdot \varphi \mathrm{d} \mathcal{H}^{1}-\int_{\Omega} \widetilde{p} \operatorname{div} \varphi \mathrm{d} x \\
& =2 \nu \int_{\Omega} \mathbf{D}(\mathbf{u}): \mathbf{D}(\widetilde{\varphi}) \mathrm{d} x+\beta \int_{\Gamma_{1}} \mathbf{u} \cdot \widetilde{\varphi} \mathrm{d} \mathcal{H}^{1}-\int_{\Omega} p \operatorname{div} \widetilde{\varphi} \mathrm{d} x \\
& =\lambda \int_{\Gamma_{i n}} \widetilde{\varphi} \cdot \mathbf{n} \mathrm{d} \mathcal{H}^{1},
\end{aligned}
$$

the second line being obtained by change of variables, using that $\Omega=\operatorname{Ref}_{\mathcal{H}}(\Omega)$ as well as the symmetry of boundary conditions. The third line follows from $(8 a)-(8 c)$ on $\mathbf{u}$. From a similar change of variables and by symmetry of $\mathbf{n}$,

$$
\begin{aligned}
\int_{\Gamma_{i n}} \widetilde{\varphi} \cdot \mathbf{n} \mathrm{d} \mathcal{H}^{1} & =\int_{\Gamma_{i n}} \widetilde{\varphi} \cdot \widetilde{\mathbf{n}} \mathrm{d} \mathcal{H}^{1}=\int_{\Gamma_{i n}} \widetilde{\varphi \cdot \mathbf{n}} \mathrm{d} \mathcal{H}^{1} \\
& =\int_{\operatorname{Ref}_{\mathcal{H}}\left(\Gamma_{i n}\right)} \varphi \cdot \mathbf{n} \mathrm{d} \mathcal{H}^{1}=\int_{\Gamma_{i n}} \varphi \cdot \mathbf{n} \mathrm{d} \mathcal{H}^{1} .
\end{aligned}
$$


Using the same kind of arguments, $(\widetilde{\mathbf{u}}, \widetilde{p})$ also satisfies $(8 b)-(8 c)$. By uniqueness of the solution of $(8 \mathrm{a})-(8 \mathrm{c})$, we then claim that $(\widetilde{\mathbf{u}}, \widetilde{p})=(\mathbf{u}, p)$. This shows the first claim of the proposition.

Now, since $u_{2} \in H^{1}(\Omega)$ and according to the symmetry property we have just proved, one has $u_{2}=-u_{2}$ a.e. on $\Gamma_{\text {sym }}$, and thus

$$
\mathbf{u} \cdot \mathbf{n}=u_{2}=0 \text { on } \Gamma_{\text {sym }} .
$$

Denoting $f=\sigma(\mathbf{u}, p) \mathbf{e}_{2}$ and since $f \in H^{1}\left(\Omega, \mathbb{R}^{2}\right)$, one has,

$$
\begin{aligned}
f \circ \operatorname{Ref}_{\mathcal{H}} & =\left(\begin{array}{c}
\nu\left(\partial_{2} u_{1} \circ \operatorname{Ref}_{\mathcal{H}}+\partial_{1} u_{2} \circ \operatorname{Ref}_{\mathcal{H}}\right) \\
2 \nu \partial_{2} u_{2} \circ \operatorname{Ref}_{\mathcal{H}}-p \circ \operatorname{Ref}_{\mathcal{H}}
\end{array}\right) \\
& =\left(\begin{array}{c}
-\nu\left(\partial_{2} u_{1}+\partial_{1} u_{2}\right) \\
2 \nu \partial_{2} u_{2}-p
\end{array}\right)=-\operatorname{Ref}_{\mathcal{H}} \circ f .
\end{aligned}
$$

Projecting this equality on axis $\mathbf{e}_{1}$, we obtain $f \cdot \mathbf{e}_{1}=-f \cdot \mathbf{e}_{1}$ a.e. on $\Gamma_{\text {sym }}$. We then infer that

$$
[\sigma(\mathbf{u}, p) \mathbf{n}]_{\tau}=0 \text { on } \Gamma_{\text {sym }} .
$$

\section{Proof of Theorem 2.2}

Let us first recall some convergence and topological notions for the elements of $\mathcal{O}_{\text {ad }}$.

Definition D.1. Recall that $D$ denotes a given compact set. A sequence of open domains $\left(\Omega_{n}\right)_{n \geq 0}$ compactly embedded in $D$ is said

- converging to $\Omega$ for the Hausdorff convergence if

$$
\lim _{n \rightarrow+\infty} d_{\mathcal{H}}\left(D \backslash \Omega_{n}, D \backslash \Omega\right)=0,
$$

where $d_{\mathcal{H}}\left(K_{1}, K_{2}\right)=\max \left(\rho\left(K_{1}, K_{2}\right), \rho\left(K_{2}, K_{1}\right)\right)$, for any $(i, j) \in\{1,2\}^{2}, \rho\left(K_{i}, K_{j}\right)=$ $\sup _{x \in K_{i}} d\left(x, K_{j}\right)$, and $\forall x \in D, d\left(x, K_{i}\right)=\inf _{y \in K_{i}} d(x, y)$;

- converging to $\Omega$ in the sense of characteristic functions if for all $p \in[1,+\infty)$,

$$
\chi_{\Omega_{n}} \underset{n \rightarrow \infty}{\longrightarrow} \chi_{\Omega} \text { in } L_{l o c}^{p}\left(\mathbb{R}^{2}\right) ;
$$

- converging to $\Omega$ in the sense of compacts if

1. $\forall K$ compact subset of $D, K \subset \Omega \Rightarrow \exists n_{0} \in \mathbb{N}^{*}, \forall n \geq n_{0}, K \subset \Omega_{n}$;

2. $\forall K$ compact subset of $D, K \subset D \backslash \bar{\Omega} \Rightarrow \exists n_{0} \in \mathbb{N}^{*}, \forall n \geq n_{0}, K \subset D \backslash \overline{\Omega_{n}}$.

We first stress that the class of admissible domains is closed and compact at the same time for the Hausdorff topology, the convergence of characteristic functions and in the sense of compacts. Indeed, this is a direct consequence of stability with respect to inclusion for the Hausdorff topology, as well as the closure of the set of domains satisfying the $\varepsilon$-cone condition for the three aforementioned topologies.

Let $\left(\Omega_{n}\right)_{n \in \mathbb{N}}$ be a minimizing sequence for Problem (20). Since the open sets $\Omega_{n}$ are contained in a fixed compact set $D$, there exists a subsequence, still denoted (with a slight abuse of notation) by $\Omega_{n}$ converging (for the Hausdorff distance, but also for the other usual topologies) to some set $\Omega$. Moreover, according to the remark above about the 
closure of admissible sets, $\Omega$ belongs to the class $\mathcal{O}_{a d}, \Omega \subset D$ and $\Omega$ satisfies the $\varepsilon$-cone property (see e.g. [29, Theorem 2.4.10]).

To prove the existence result, it remains to show the lower-semicontinuity of the criterion $J$. For every $n \in \mathbb{N}$, we denote by $\left(\mathbf{u}_{\mathbf{n}}, p_{n}, \lambda_{n}\right) \in V\left(\Omega_{n}\right) \times L^{2}\left(\Omega_{n}\right) \times \mathbb{R}$ the unique triple satisfying (8a)-(8b)-(8c) (see Proposition 1.3).

Since $\left(\Omega_{n}\right)_{n \in \mathbb{N}}$ is a minimizing sequence for Problem (20), we infer that the sequence

$$
\left(\max \left\{\int_{\Omega_{n}}\left|\mathbf{D}\left(\mathbf{u}_{n}\right)\right|^{2} \mathrm{~d} x, \int_{\Gamma_{1}^{n}}\left|\mathbf{u}_{n}\right|^{2} \mathrm{~d} \mathcal{H}^{1}\right\}\right)_{n \in \mathbb{N}},
$$

with $\Gamma_{1}^{n}=\partial \Omega_{n} \backslash\left(\Gamma_{\text {in }} \cup \Gamma_{0} \cup \Gamma_{2} \cup \Gamma_{\text {out }}\right)$, is bounded.

This shows that, up to subsequences, $\left(\mathbf{u}_{\mathbf{n}} \mathbb{1}_{\Omega_{n}}\right)$ converges weakly to some function $\mathbf{v} \in L^{2}\left(D, \mathbb{R}^{2}\right)$ whereas $\left(\mathbf{D}\left(\mathbf{u}_{\mathbf{n}}\right) \mathbb{1}_{\Omega_{n}}\right)$ converges weakly to some function $\mathbf{z} \in L^{2}\left(D, \mathcal{S}_{2}(\mathbb{R})\right)$ (the notation $\mathcal{S}_{2}(\mathbb{R})$ denoting the set of real-symmetric matrices of $\mathbb{R}^{2}$ ).

Let us show the existence of $\mathbf{u} \in H^{1}\left(\Omega, \mathbb{R}^{2}\right)$ such that $\mathbf{v}=\mathbf{u} \mathbb{1}_{\Omega}$ and $\mathbf{z}=\mathbf{D}(\mathbf{u}) \mathbb{1}_{\Omega}$. For every $\varphi \in L^{2}\left(D, \mathbb{R}^{2}\right)$, one has

$$
\int_{D} \mathbf{u}_{\mathbf{n}} \mathbb{1}_{\Omega_{n}} \cdot \varphi \mathrm{d} x \underset{n \rightarrow+\infty}{\longrightarrow} \int_{D} \mathbf{v} \mathbb{1}_{\Omega} \cdot \varphi \mathrm{d} x=\int_{D} \mathbf{v} \cdot \varphi \mathrm{d} x,
$$

and therefore, $\mathbf{v}=\mathbf{v} \mathbb{1}_{\Omega}$. Similarly, for every $\psi \in L^{2}\left(D, \mathcal{M}_{d}(\mathbb{R})\right)$, one has

$$
\int_{D} \mathbf{D}\left(\mathbf{u}_{\mathbf{n}}\right) \mathbb{1}_{\Omega_{n}}: \varphi \mathrm{d} x \underset{n \rightarrow+\infty}{\longrightarrow} \int_{D} \mathbf{z} \mathbb{1}_{\Omega} \cdot \varphi \mathrm{d} x=\int_{D} \mathbf{z}: \psi \mathrm{d} x
$$

so that $\mathbf{z}=\mathbf{z} \mathbb{1}_{\Omega}$. Let $\mathbf{u}$ be the restriction of $\mathbf{v}$ to $\Omega$, one has for all $(i, j) \in\{1,2\}^{2}$ and $\varphi \in C_{c}^{\infty}(\bar{\Omega})$,

$$
\begin{aligned}
\lim _{n \rightarrow+\infty} \int_{D} \mathbb{1}_{\Omega_{n}} u_{n, i} \frac{\partial \varphi}{\partial x_{j}} \mathrm{~d} x & =\int_{D} \mathbb{1}_{\Omega} u_{i} \frac{\partial \varphi}{\partial x_{j}} \mathrm{~d} x \\
& =-\lim _{n \rightarrow+\infty} \int_{D} \mathbb{1}_{\Omega_{n}} \varphi \frac{\partial u_{n, i}}{\partial x_{j}} \mathrm{~d} x=-\int_{D} \mathbb{1}_{\Omega} \varphi z_{i j} \mathrm{~d} x
\end{aligned}
$$

by using the Green formula and that $\varphi \in C_{c}^{\infty}\left(\overline{\Omega_{n}}\right)$ for $n$ large enough. As a consequence, there holds $\mathbf{z}=\nabla \mathbf{u} \mathbb{1}_{\Omega}$, showing the weak convergence of $\left(\mathbf{D}\left(\mathbf{u}_{\mathbf{n}}\right) \mathbb{1}_{\Omega_{n}}\right)_{n \in \mathbb{N}}$ to $\mathbf{D}(\mathbf{u}) \mathbb{1}_{\Omega}$.

To prove the strong convergence of $\left(\mathbf{u}_{\mathbf{n}} \mathbb{1}_{\Omega_{n}}\right)_{n \in \mathbb{N}}$ in $L^{2}\left(D, \mathbb{R}^{2}\right)$ to $\mathbf{u} \mathbb{1}_{\Omega}$ as well as the semicontinuity of the boundary term of $J$, we will adapt [10, Lemma 4.2].

This way, we only underline the slight changes needed to get the result. The first step consists in investigating the behaviour of the minimizing sequence on subdomains and contains the main differences with [10, Lemma 4.2]. Hence, let us consider a subset $\hat{\Omega}$ having a compact closure in $\Omega$ and a Lipschitz boundary. Using the convergence in the sense of compacts, we know that $\hat{\Omega} \subset \Omega_{n} \subset \Omega$ for $n$ large enough. Therefore, the function $\mathbf{u}_{\mathbf{n}}$ belongs to $H^{1}\left(\hat{\Omega}, \mathbb{R}^{2}\right)$ for $n$ large enough. By using the Rellich-Kondratov embedding theorem, one infers that $\left(\mathbf{u}_{\mathbf{n}}\right)_{n \in \mathbb{N}}$ converges strongly in $L^{2}\left(\hat{\Omega}, \mathbb{R}^{2}\right)$ and weakly in $H^{1}\left(\hat{\Omega}, \mathbb{R}^{2}\right)$ to $\mathbf{u}$. This follows in particular from the Korn inequality in $\hat{\Omega}$ which asserts that the usual $H^{1}$-norm is equivalent to the norm $\|\cdot\|_{L^{2}}+\|\mathbf{D}(\cdot)\|_{L^{2}}$, since there exists a subset of $\partial \Omega$ of positive Hausdorff measure (namely $\Gamma_{0}$ ) on which homogeneous Dirichlet boundary conditions are imposed.

To conclude, it remains to investigate the global behaviour of the sequence on the whole domain. By using the strong convergence on subdomains $\hat{\Omega}$ we have just proved, 
the proof consists of a direct adaptation of [10, Proof of Lemma 4.2]. For this reason, we refer to [10, Proof of Lemma 4.2] and infer successively that $\left(\mathbf{u}_{\mathbf{n}} \mathbb{1}_{\Omega_{n}}\right)_{n \in \mathbb{N}}$ converges strongly to $\mathbf{u}_{\Omega}$ in $L^{2}\left(D, \mathbb{R}^{2}\right)$ and

$$
\int_{\Gamma_{1}}|\mathbf{u}|^{2} \mathrm{~d} \mathcal{H}^{1} \leq \liminf _{n \rightarrow+\infty} \int_{\Gamma_{1}^{n}}\left|\mathbf{u}_{\mathbf{n}}\right|^{2} \mathrm{~d} \mathcal{H}^{1}
$$

with $\Gamma_{1}^{n}=\partial \Omega_{n} \backslash\left(\Gamma_{\text {in }} \cup \Gamma_{0} \cup \Gamma_{2} \cup \Gamma_{\text {out }}\right)$ and $\Gamma_{1}=\partial \Omega \backslash\left(\Gamma_{\text {in }} \cup \Gamma_{0} \cup \Gamma_{2} \cup \Gamma_{\text {out }}\right)$.

Combining the previous results, we then infer that (up to subsequences)

$$
\mathcal{E}_{\Omega}(\mathbf{u}) \leq \liminf _{n \rightarrow+\infty} J\left(\Omega_{n}\right) .
$$

To conclude, it remains to show that $\mathbf{u}$ belongs to the space $V_{\text {div }}(\Omega)$ (defined by (10)). Notice first that, on fixed boundaries, one has obviously $\mathbf{u}_{\mid \Gamma_{0}}=0, \mathbf{u}_{\mid \Gamma_{2}}=0$ and $\int_{\Gamma_{i n}} \mathbf{u}$. $\mathbf{n} \mathrm{d} \mathcal{H}^{1}=-Q$ (the last equality following from the weak $H^{1}$-convergence of the sequence $\left(\mathbf{u}_{\mathbf{n}}\right)_{n \in \mathbb{N}}$ in a neighborhood of $\Gamma_{i n}$ in $\Omega$ combined with the trace continuity property on $\left.\Gamma_{i n}\right)$. It remains to show that

$$
\operatorname{div} \mathbf{u}=0 \quad \text { in } \Omega \quad \text { and } \quad \mathbf{u} \cdot \mathbf{n}=0 \quad \text { on } \Gamma_{1} .
$$

Using an integration by parts, these two conditions can be gathered under the weak form

$$
\int_{\Omega} \mathbf{u} \cdot \nabla \varphi \mathrm{d} x=0
$$

for every test function $\varphi \in H^{1}(D)$ such that $\varphi=0$ on $\Gamma_{i n} \cup \Gamma_{0} \cup \Gamma_{2} \cup \Gamma_{\text {out }}$. This is obtained by passing to the limit in the equality

$$
\int_{D} \mathbb{1}_{\Omega_{n}} \mathbf{u}_{\mathbf{n}} \cdot \nabla \varphi \mathrm{d} x=0
$$

where $\mathbf{u}_{\mathbf{n}}$ has been extended by 0 to the whole compact set $D$ and $\varphi$ denotes any test function in $H^{1}(D)$ such that $\varphi=0$ on $\Gamma_{\text {in }} \cup \Gamma_{0} \cup \Gamma_{2} \cup \Gamma_{\text {out }}$.

\section{E Proof of Proposition 2.3}

First step: differentiability of $J$ and $u$ with respect to the domain. Differentiability of volumic criteria with respect to domain variations is generally proved by using standard technics resting upon the implicit function theorem (see e.g. [4] and [29, Theorems 5.3.1 and 5.3.2]). In the case that we investigate, we have to take into account particular boundary conditions, namely the slip boundary conditions, which requires several adaptations. For this reason, we prove the shape-differentiability of $J$ in detail. According to the proof of Proposition 1.3, the solution $\mathbf{u}_{\lambda}$ to problem (8a)-(8c) reads $\mathbf{u}_{\lambda}=\lambda \mathbf{u}_{1}$, where $\mathbf{u}_{1}$ is the solution to (32)-(33) and $\lambda$ is given by (34). Consequently, it is enough to prove the differentiability of $\mathbf{u}_{1}$ with respect to domain variations to conclude that $\lambda$ and $\mathbf{u}_{\lambda}$ are differentiable as well.

Let $\left(\mathbf{u}_{1, t}, p_{1, t}\right) \in V\left(\Omega_{t}\right) \times L^{2}\left(\Omega_{t}\right)$ be the unique solution to

$$
\begin{aligned}
& \forall \varphi \in V\left(\Omega_{t}\right) \quad 2 \nu \int_{\Omega_{t}} \mathbf{D}\left(\mathbf{u}_{1, t}\right): \mathbf{D}(\varphi) \mathrm{d} x+\beta \int_{\Phi_{t}\left(\Gamma_{1}\right)} \mathbf{u}_{1, t} \cdot \varphi \mathrm{d} \mathcal{H}^{1} \\
& \quad-\int_{\Omega_{t}} p_{1, t} \operatorname{div} \varphi \mathrm{d} x=\int_{\Gamma_{i n}} \varphi \cdot \mathbf{n} \mathrm{d} \mathcal{H}^{1}, \\
& \operatorname{div} \mathbf{u}_{1, t}=0 \quad \text { in } \Omega_{t} .
\end{aligned}
$$


To address the differentiability of $\left(\mathbf{u}_{1, t}, p_{1, t}\right)$ with respect to $t$, we need to recast problem (39)-(40) into the reference domain $\Omega$, by introducing a change of function performed on both the solution $\left(\mathbf{u}_{1, t}, p_{1, t}\right)$ and the test functions $(\varphi, q)$.

Let $t \in(-T, T)$ be fixed. We denote by $J_{t}(y)=D_{y} \Phi_{t}(y)$ the Jacobian matrix of $\Phi_{t}$ at point $y$, by $\mathcal{J}_{t}(y)$ its determinant and we define $M_{t}(y)=J_{t}(y)^{-1}$. We introduce the function $R_{t} \in L^{2}(\Omega)$ defined by $R_{t}=\mathcal{J}_{t} p_{1, t} \circ \Phi_{t}$. Since $p_{1, t} \in L^{2}\left(\Omega_{t}\right)$, using the change of variable associated with $\Phi_{t}$, we see that $R_{t} \in L^{2}(\Omega)$. Now, consider the function $\mathbf{U}_{t} \in H^{1}(\Omega)$ defined by

$$
\mathbf{U}_{t}=M_{t}\left(\mathbf{u}_{1, t} \circ \Phi_{t}\right) .
$$

The product with the matrix $M_{t}$ is justified by the fact that the new function $\mathbf{U}_{t}$ satisfies the non penetration condition $\mathbf{U}_{t} \cdot \mathbf{n}=0$ on $\Gamma_{1}$ (see, for instance, [7]). Consequently, by construction of the diffeomorphism $\Phi_{t}, \mathbf{U}_{t} \in V\left(\Omega_{t}\right)$. The Jacobian matrix $\nabla \mathbf{u}_{1, t}$ and the divergence div $\mathbf{u}_{1, t}$ are transformed as follows:

$$
\nabla \mathbf{u}_{1, t} \circ \Phi_{t}=\left(H_{t} \mathbf{U}_{t}+J_{t} \nabla \mathbf{U}_{t}\right) M_{t}, \quad\left(\operatorname{div} \mathbf{u}_{1, t}\right) \circ \Phi_{t}=\operatorname{Tr}\left[\left(H_{t} \mathbf{U}_{t}+J_{t} \nabla \mathbf{U}_{t}\right) M_{t}\right]
$$

where $\operatorname{Tr}(A)$ is the trace of a square matrix $A$, and for any $\mathbf{U} \in V(\Omega), H_{t} \mathbf{U} \in H^{1}\left(\Omega, \mathbb{R}^{2 \times 2}\right)$ is defined component by component by $\left(H_{t} \mathbf{U}\right)_{i, j}=\sum_{k=1}^{d} \frac{\partial^{2} \Phi_{i}(t)}{\partial y_{j} \partial y_{k}} U_{k}$.

For every $\varphi \in V\left(\Omega_{t}\right)$, following the previous remarks, we can define $\tilde{\varphi} \in V(\Omega)$ by $\tilde{\varphi}=M_{t} \varphi \circ \Phi_{t}$. Problem (39)-(40) is then equivalent to

$$
\begin{array}{r}
\forall \tilde{\varphi} \in V(\Omega) \quad \frac{\nu}{2} \int_{\Omega}\left(\left(H_{t} \mathbf{U}_{t}+J_{t} \nabla \mathbf{U}_{t}\right) M_{t}+\left[\left(H_{t} \mathbf{U}_{t}+J_{t} \nabla \mathbf{U}_{t}\right) M_{t}\right]^{T}\right): \\
\left(\left(H_{t} \tilde{\varphi}+J_{t} \nabla \tilde{\varphi}\right) M_{t}+\left[\left(H_{t} \tilde{\varphi}+J_{t} \nabla \tilde{\varphi}\right) M_{t}\right]^{T}\right) J_{t} \mathrm{~d} y \\
+\beta \int_{\Gamma_{1}}\left(J_{t} \mathbf{U}_{t}\right) \cdot\left(J_{t} \tilde{\varphi}\right)\left|M_{t}^{T} \mathbf{n}\right| \mathcal{J}_{t} \mathrm{~d} \mathcal{H}^{1}-\int_{\Omega} R_{t} \operatorname{Tr}\left[\left(H_{t} \tilde{\varphi}+J_{t} \nabla \tilde{\varphi}\right) M_{t}\right] \mathrm{d} y \\
=\int_{\Gamma_{i n}} \tilde{\varphi} \cdot \mathbf{n} \mathrm{d} \mathcal{H}^{1}, \\
\operatorname{Tr}\left[\left(H_{t} \mathbf{U}_{t}+J_{t} \nabla \mathbf{U}_{t}\right) M_{t}\right]=0 \quad \text { in } \Omega .
\end{array}
$$

In view of equations (41)-(42), we introduce the operator

$$
\begin{aligned}
F:(-T, T) \times V(\Omega) \times L^{2}(\Omega) & \rightarrow[V(\Omega)]^{\prime} \times L^{2}(\Omega) \\
(t, \mathbf{U}, R) & \mapsto\left(F_{1}(t, \mathbf{U}, R), F_{2}(t, \mathbf{U}, R)\right)
\end{aligned}
$$

where for all $\tilde{\varphi} \in V(\Omega)$, one has

$$
\begin{aligned}
\left\langle F_{1}(t, \mathbf{U}, R), \tilde{\varphi}\right\rangle_{[V(\Omega)]^{\prime} \times V(\Omega)}= & \frac{\nu}{2} \int_{\Omega}\left(\left(H_{t} \mathbf{U}+J_{t} \nabla \mathbf{U}\right) M_{t}+\left[\left(H_{t} \mathbf{U}+J_{t} \nabla \mathbf{U}\right) M_{t}\right]^{T}\right): \\
& \left(\left(H_{t} \tilde{\varphi}+J_{t} \nabla \tilde{\varphi}\right) M_{t}+\left[\left(H_{t} \tilde{\varphi}+J_{t} \nabla \tilde{\varphi}\right) M_{t}\right]^{T}\right) J_{t} \mathrm{~d} y \\
& +\beta \int_{\Gamma_{1}}\left(J_{t} \mathbf{U}\right) \cdot\left(J_{t} \tilde{\varphi}\right)\left|M_{t}^{T} \mathbf{n}\right| \mathcal{J}_{t} \mathrm{~d} \mathcal{H}^{1} \\
& -\int_{\Omega} R \operatorname{Tr}\left[\left(H_{t} \tilde{\varphi}+J_{t} \nabla \tilde{\varphi}\right) M_{t}\right] \mathrm{d} y-\int_{\Gamma_{i n}} \tilde{\varphi} \cdot \mathbf{n} \mathrm{d} \mathcal{H}^{1}, \\
F_{2}(t, \mathbf{U}, R)= & \operatorname{Tr}\left[\left(H_{t} \mathbf{U}+J_{t} \nabla \mathbf{U}\right) M_{t}\right] .
\end{aligned}
$$

For every $t \in(-T, T)$, by uniqueness of the solution $\left(\mathbf{u}_{1, t}, p_{1, t}\right) \in V\left(\Omega_{t}\right) \times L^{2}\left(\Omega_{t}\right)$ to (39)(40), there exists a unique pair $\left(\mathbf{U}_{t}, R_{t}\right) \in V(\Omega) \times L^{2}(\Omega)$ such that $F\left(t, \mathbf{U}_{t}, R_{t}\right)=0$. 
We will apply the implicit function theorem to prove that the mapping $t \mapsto\left(\mathbf{U}_{t}, R_{t}\right)$ is differentiable at $t=0$. Since the mapping $t \in(-T, T) \mapsto \Phi_{t} \in W^{2, \infty}\left(\mathbb{R}^{2}, \mathbb{R}^{2}\right)$ is of class $\mathcal{C}^{1}$, every coefficient appearing in the operator $H_{t}$ and the matrices $J_{t}, M_{t}$ is of class $\mathcal{C}^{1}$ in $t$. Consequently, $F$ is of class $\mathcal{C}^{1}$ with respect to $(t, \mathbf{U}, R)$, and its differential with respect to $(\mathbf{U}, R)$ at point $\left(0, \mathbf{U}_{0}, R_{0}\right)$ reads

$$
\begin{gathered}
\forall(\mathbf{Z}, S) \in V(\Omega) \times L^{2}(\Omega) \quad \forall \varphi \in V(\Omega) \\
\left\langle D_{(\mathbf{U}, R)} F_{1}\left(0, \mathbf{U}_{0}, R_{0}\right)(\mathbf{Z}, S), \varphi\right\rangle=2 \nu \int_{\Omega} \mathbf{D}(\mathbf{Z}): \mathbf{D}(\varphi) \mathrm{d} y \\
+\beta \int_{\Gamma_{1}} \mathbf{Z} \cdot \varphi \mathrm{d} \mathcal{H}^{1}-\int_{\Omega} S \operatorname{div} \varphi \mathrm{d} y, \\
D_{(\mathbf{U}, R)} F_{2}\left(0, \mathbf{U}_{0}, R_{0}\right)(\mathbf{Z}, S)=\operatorname{div} \mathbf{Z} .
\end{gathered}
$$

Let us prove that $D_{(\mathbf{U}, R)} F\left(0, \mathbf{U}_{0}, R_{0}\right) \in \mathcal{L}\left(V(\Omega) \times L^{2}(\Omega),[V(\Omega)]^{\prime} \times L^{2}(\Omega)\right)$ is an isomorphism. To this end, consider $(\mathbf{G}, s) \in[V(\Omega)]^{\prime} \times L^{2}(\Omega)$. Since $V(\Omega)$ is a Hilbert space for the scalar product $(\mathbf{U}, \varphi) \in V(\Omega) \times V(\Omega) \mapsto \int_{\Omega} \nabla \mathbf{U}: \nabla \varphi \mathrm{d} x$, by Riesz theorem we can identify $\mathbf{G}$ with its representative in $V(\Omega)$, and define for every $\varphi \in V(\Omega)$ the duality pairing

$$
\langle\mathbf{G}, \varphi\rangle_{[V(\Omega)]^{\prime}, V(\Omega)}:=\int_{\Omega} \nabla \mathbf{G}: \nabla \varphi \mathrm{d} x .
$$

By lemma A.1, there exists a constant $C>0$ and a function $\mathbf{v} \in V(\Omega)$ such that $\operatorname{div} \mathbf{v}=s$ a.e. in $\Omega$ and $\|\mathbf{v}\|_{V(\Omega)} \leq C\|s\|_{L^{2}(\Omega)}$. Now, define $\left(\mathbf{Z}_{0}, S\right) \in V(\Omega) \times L^{2}(\Omega)$ as the unique solution to the following problem:

$$
\begin{aligned}
\forall \varphi \in V(\Omega) \quad & 2 \nu \int_{\Omega} \mathbf{D}\left(\mathbf{Z}_{0}\right): \mathbf{D}(\varphi) \mathrm{d} y+\beta \int_{\Gamma_{1}} \mathbf{Z}_{0} \cdot \varphi \mathrm{d} \mathcal{H}^{1}-\int_{\Omega} S \operatorname{div} \varphi \mathrm{d} y \\
= & \int_{\Omega} \nabla \mathbf{G}: \nabla \varphi \mathrm{d} x-2 \nu \int_{\Omega} \mathbf{D}(\mathbf{v}): \mathbf{D}(\varphi) \mathrm{d} y-\beta \int_{\Gamma_{1}} \mathbf{v} \cdot \varphi \mathrm{d} \mathcal{H}^{1}, \\
\operatorname{div} \mathbf{Z}_{0}=0 & \text { in } \Omega .
\end{aligned}
$$

By classical arguments, there exists a constant $C>0$ such that

$$
\left\|\mathbf{Z}_{0}\right\|_{V(\Omega)} \leq C\left(\|\mathbf{G}\|_{V(\Omega)}+\|\mathbf{v}\|_{H^{1}\left(\Omega, \mathbb{R}^{2}\right)}\right),
$$

and in view of the previous estimates and Poincaré inequaity,

$$
\left\|\mathbf{Z}_{0}\right\|_{V(\Omega)} \leq C\left(\|\mathbf{G}\|_{V(\Omega)}+\|s\|_{L^{2}\left(\Omega, \mathbb{R}^{2}\right)}\right) .
$$

Finally, define $\mathbf{Z} \in V(\Omega)$ by $\mathbf{Z}=\mathbf{Z}_{0}+\mathbf{v}$. Then, the pair $(\mathbf{Z}, S)$ is the unique solution to the problem

$$
D_{(\mathbf{U}, R)} F\left(0, \mathbf{U}_{0}, R_{0}\right)(\mathbf{Z}, S)=(\mathbf{G}, s),
$$

and satisfies the estimate

$$
\|\mathbf{Z}\|_{V(\Omega)}+\|S\|_{L^{2}(\Omega)} \leq C\left(\|\mathbf{G}\|_{V(\Omega)}+\|s\|_{L^{2}\left(\Omega, \mathbb{R}^{2}\right)}\right) .
$$

By the implicit function theorem, there exists $T_{0}>0$ such that the mapping $t \in$ $\left(-T_{0}, T_{0}\right) \mapsto\left(\mathbf{U}_{t}, R_{t}\right) \in V(\Omega) \times L^{2}(\Omega)$ is differentiable, and since the mapping $t \mapsto J_{t}$ is regular, we deduce that the mapping $t \in\left(-T_{0}, T_{0}\right) \mapsto\left(\mathbf{u}_{1, t} \circ \Phi_{t}, p_{1, t} \circ \Phi_{t}\right) \in V(\Omega) \times L^{2}(\Omega)$ is differentiable. 
Second step: computation of the shape derivative. Assume that $\partial \Omega$ is of class $\mathcal{C}^{2}$. Notice that such assumption yield the existence of strong solutions of the involved partial differential equations. In particular, System (11) has a unique solution $\left(\mathbf{u}_{\lambda}, p_{\lambda}, \lambda\right)$ belonging to the space $\left[V(\Omega) \cap H^{2}\left(\Omega, \mathbb{R}^{2}\right)\right] \times H^{1}(\Omega) \times \mathbb{R}$.

Let $\mathbf{V} \in W^{3, \infty}\left(\mathbb{R}^{2}, \mathbb{R}^{2}\right)$. We introduce $T>0$ and a mapping $t \in(-T, T) \mapsto \Phi_{t} \in$ $W^{2, \infty}\left(\mathbb{R}^{2}, \mathbb{R}^{2}\right)$, of class $\mathcal{C}^{3}$, satisfying $\Phi_{0}=\mathrm{Id}$ and $\frac{d \Phi_{t}}{d_{t}}{ }_{t=0}=\mathbf{V}$. Assume moreover that $\Phi_{t}(x)=x$ for every $x \in \partial \Omega \backslash \Gamma_{1}$ and every $t$. We set $\Omega_{t}:=\Phi_{t}(\Omega)$ and define the shape derivative of $J$ at $\Omega$, in the direction $\mathbf{V}$, by

$$
\langle d J(\Omega), \mathbf{V}\rangle=\lim _{t \searrow 0} \frac{J\left(\Omega_{t}\right)-J(\Omega)}{t} .
$$

Let us denote by $\left(\mathbf{u}_{\lambda}^{\prime}, p_{\lambda}^{\prime}\right)$ the Eulerian derivative of the pair $\left(\mathbf{u}_{\lambda}, p_{\lambda}\right)$, in other words the derivative of the mapping $t \mapsto\left(\mathbf{u}_{\lambda, t}, p_{\lambda, t}\right) \in H^{1}(\Omega) \times L^{2}(\Omega)$ at $t=0$. It is rather standard to differentiate the partial differential equation (11) with respect to the domain perturbation. To that end, we need to introduce an extension of the normal vector. Recall that the final expression of the shape derivatives does not depend on the choice of extension (see [29, Theorem 5.9.2]).

Let us consider a symmetric extension $\mathbf{n}_{t}$ of the normal on $\partial \Omega_{t}$, in other words such that $\nabla \mathbf{n}_{t}$ is a symmetric matrix a.e. in $\overline{\Omega_{t}}$. Then the Eulerian derivative of this extension is given by

$$
\frac{\partial \mathbf{n}_{t}}{\partial t}=\mathbf{n}^{\prime}=-\nabla_{\Gamma}(\mathbf{V} \cdot \mathbf{n})
$$

where $\nabla_{\Gamma}$ is the tangential gradient operator and $\mathbf{n}^{\prime}$ stands for the derivative of $t \mapsto \mathbf{n}_{t}$ at $t=0$.

Using classical shape derivation rules [29, Sections 5.6 and 5.8], one gets

$$
\begin{aligned}
\langle d J(\Omega), \mathbf{V}\rangle & =\int_{\Gamma_{1}}\left[2 \nu\left|\mathbf{D}\left(\mathbf{u}_{\lambda}\right)\right|^{2}+\beta\left(H\left|\mathbf{u}_{\lambda}\right|^{2}+\partial_{n}\left(\left|\mathbf{u}_{\lambda}\right|^{2}\right)\right)\right](\mathbf{V} \cdot \mathbf{n}) \mathrm{d} \mathcal{H}^{1} \\
& +\int_{\Omega} 4 \nu \mathbf{D}\left(\mathbf{u}_{\lambda}\right): \mathbf{D}\left(\mathbf{u}_{\lambda}^{\prime}\right) d x+\int_{\Gamma_{1}} 2 \beta \mathbf{u}_{\lambda} \cdot \mathbf{u}_{\lambda}^{\prime} \mathrm{d} \mathcal{H}^{1},
\end{aligned}
$$

where $H$ denotes the mean curvature on $\partial \Omega$.

In order to get a more workable expression of this quantity (in view of numerical simulations), the general method is to introduce an adjoint problem to rewrite the term $4 \nu \int_{\Omega} \mathbf{D}\left(\mathbf{u}_{\lambda}\right): \mathbf{D}\left(\mathbf{u}_{\lambda}^{\prime}\right) \mathrm{d} x+2 \beta \int_{\Gamma_{1}} \mathbf{u}_{\lambda} \cdot \mathbf{u}_{\lambda}^{\prime} \mathrm{d} \mathcal{H}^{1}$ under the form $\int_{\Gamma} G(\mathbf{V} \cdot \mathbf{n}) \mathrm{d} \mathcal{H}^{1}$, where $G$ does not depend on $\mathbf{V}$. However, since the criterion $J(\Omega)$ can be rewritten as the minimum of an energy functional, this problem is in some sense self-adjoint, meaning that the shape derivative can be expressed in terms of $\mathbf{u}_{\lambda}$ and $p_{\lambda}$ only.

In order to eliminate the last line from (44), let us use equation (11) and integrate by 
parts taking $\mathbf{u}_{\lambda}^{\prime}$ as a test-function. We obtain

$$
\begin{aligned}
0=\int_{\Omega}-\operatorname{div}\left(\sigma\left(\mathbf{u}_{\lambda}, p_{\lambda}\right)\right) \cdot \mathbf{u}_{\lambda}^{\prime} d x= & \int_{\Omega} 2 \nu \mathbf{D}\left(\mathbf{u}_{\lambda}\right): \mathbf{D}\left(\mathbf{u}_{\lambda}^{\prime}\right) d x-\int_{\partial \Omega} \sigma\left(\mathbf{u}_{\lambda}, p_{\lambda}\right) \mathbf{n} \cdot \mathbf{u}_{\lambda}^{\prime} \mathrm{d} \mathcal{H}^{1} \\
= & \int_{\Omega} 2 \nu \mathbf{D}\left(\mathbf{u}_{\lambda}\right): \mathbf{D}\left(\mathbf{u}_{\lambda}^{\prime}\right) d x-\int_{\Gamma_{1}} \sigma\left(\mathbf{u}_{\lambda}, p_{\lambda}\right) \mathbf{n} \cdot \mathbf{u}_{\lambda}^{\prime} \\
& -\int_{\Gamma_{i n}} \sigma\left(\mathbf{u}_{\lambda}, p_{\lambda}\right) \mathbf{n} \cdot \mathbf{u}_{\lambda}^{\prime} \\
= & \int_{\Omega} 2 \nu \mathbf{D}\left(\mathbf{u}_{\lambda}\right): \mathbf{D}\left(\mathbf{u}_{\lambda}^{\prime}\right) d x+\int_{\Gamma_{1}} \beta \mathbf{u}_{\lambda} \cdot \mathbf{u}_{\lambda}^{\prime} \\
& -\int_{\Gamma_{1}}\left(\sigma\left(\mathbf{u}_{\lambda}, p_{\lambda}\right) \mathbf{n} \cdot \mathbf{n}\right)\left(\mathbf{u}_{\lambda}^{\prime} \cdot \mathbf{n}\right)-\lambda \int_{\Gamma_{i n}} \mathbf{u}_{\lambda}^{\prime} \cdot \mathbf{n} \mathrm{d} \mathcal{H}^{1} .
\end{aligned}
$$

From the equality

$$
\int_{\Gamma_{\text {in }}} \mathbf{u}_{\lambda} \cdot \mathbf{n} \mathrm{d} \mathcal{H}^{1}=-Q
$$

we infer (with a slight abuse of notation)

$$
\left\langle d \int_{\Gamma_{i n}} \mathbf{u}_{\lambda} \cdot \mathbf{n} \mathrm{d} \mathcal{H}^{1}, \mathbf{V}\right\rangle=\int_{\Gamma_{i n}} \mathbf{u}_{\lambda}^{\prime} \cdot \mathbf{n} \mathrm{d} \mathcal{H}^{1}=0 .
$$

Finally, on $\Gamma_{1}$, one has

$$
\mathbf{u}_{\lambda}^{\prime} \cdot \mathbf{n}=-\partial_{n}\left(\mathbf{u}_{\lambda} \cdot \mathbf{n}\right)(\mathbf{V} \cdot \mathbf{n})+\mathbf{u}_{\lambda} \cdot \nabla_{\tau}(\mathbf{V} \cdot \mathbf{n}) .
$$

After rearranging the terms, one finally gets

$$
\begin{aligned}
\langle d J(\Omega), \boldsymbol{\theta}\rangle & =\int_{\Gamma_{1}}\left[2 \nu\left|\mathbf{D}\left(\mathbf{u}_{\lambda}\right)\right|^{2}+\beta\left(H\left|\mathbf{u}_{\lambda}\right|^{2}+\partial_{n}\left(\left|\mathbf{u}_{\lambda}\right|^{2}\right)\right)-2\left(\sigma\left(\mathbf{u}_{\lambda}, p_{\lambda}\right) \mathbf{n} \cdot \mathbf{n}\right) \partial_{n}\left(\mathbf{u}_{\lambda} \cdot \mathbf{n}\right)\right](\mathbf{V} \cdot \mathbf{n}) \mathrm{d} \mathcal{H}^{1} \\
& +\int_{\Gamma_{1}} 2\left(\sigma\left(\mathbf{u}_{\lambda}, p_{\lambda}\right) \mathbf{n} \cdot \mathbf{n}\right) \mathbf{u}_{\lambda} \cdot \nabla_{\tau}(\mathbf{V} \cdot \mathbf{n}) \mathrm{d} \mathcal{H}^{1}
\end{aligned}
$$

Acknowledgements : The authors would like to warmly thank Laurent JOLY for answering several questions about modeling issues.

\section{References}

[1] G. Allaire. Conception optimale de structures, volume 58 of Mathématiques \&s Applications (Berlin) [Mathematics \& Applications]. Springer-Verlag, Berlin, 2007. With the collaboration of Marc Schoenauer (INRIA) in the writing of Chapter 8.

[2] T. J. Baker. Mesh movement and metamorphosis. Eng. Comput., 18(1):188-198, 2002.

[3] C. Belin, L. Joly, and F. Detcheverry. Optimal shape of entrances for a frictionless nanochannel. Physical Review Fluids, 1(5):054103, 2016.

[4] J. A. Bello, E. Fernandez-Cara, J. Lemoine, and J. Simon. The differentiability of the drag with respect to the variations of a lipschitz domain in a navier-stokes flow. SIAM Journal on Control and Optimization, 35(2):626-640, 1997. 
[5] G. Benga. The first discovered water channel protein, later called aquaporin 1: molecular characteristics, functions and medical implications. Molecular aspects of medicine, 33(5):518-534, 2012.

[6] L. Bocquet and J.-L. Barrat. Flow boundary conditions from nano- to micro-scales. Soft Matter, 3:685-693, 2007.

[7] M. Bonnivard. On the stability of self-propelled bodies with respect to their shape motion. Mathematical Models and Methods in Applied Sciences, 21(04):667-691, 2011.

[8] R. P. Brent. Algorithms for minimization without derivatives. Prentice Hall, 1973.

[9] D. Bucur and A. Giacomini. Shape optimization problems with Robin conditions on the free boundary. Ann. Inst. H. Poincaré Anal. Non Linéaire, 33(6):1539-1568, 2016 .

[10] D. Bucur, A. Giacomini, and P. Trebeschi. The Robin-Laplacian problem on varying domains. Calc. Var. Partial Differential Equations, 55(6):55:133, 2016.

[11] M. Burger. A framework for the construction of level set methods for shape optimization and reconstruction. Interfaces and Free Boundaries, 5(3):301-329, 2003.

[12] J. M. Carbrey and P. Agre. Discovery of the aquaporins and development of the field. In Aquaporins, pages 3-28. Springer, 2009.

[13] D. Chenais. On the existence of a solution in a domain identification problem. $J$. Math. Anal. Appl., 52(2):189-219, 1975.

[14] C. Dapogny, P. Frey, F. Omnès, and Y. Privat. GEOMETRICAL SHAPE OPTIMIZATION IN FLUID MECHANICS USING FREEFEM++. working paper or preprint, Mar. 2017.

[15] F. De Gournay. Velocity extension for the level-set method and multiple eigenvalues in shape optimization. SIAM journal on control and optimization, 45(1):343-367, 2006.

[16] X. D. de La Sablonière, B. Mauroy, and Y. Privat. Shape minimization of the dissipated energy in dyadic trees. Discrete Contin. Dyn. Syst. Ser. B, 16(3):767-799, 2011.

[17] M. C. Delfour and J.-P. Zolésio. Shapes and geometries, volume 22 of Advances in Design and Control. Society for Industrial and Applied Mathematics (SIAM), Philadelphia, PA, second edition, 2011. Metrics, analysis, differential calculus, and optimization.

[18] B. V. der Bruggen and C. Vandecasteele. Removal of pollutants from surface water and groundwater by nanofiltration: overview of possible applications in the drinking water industry. Environmental Pollution, 122(3):435 - 445, 2003.

[19] C. Dobrzynski and P. Frey. Anisotropic delaunay mesh adaptation for unsteady simulations. Proc. 17th Int. Meshing Roundtable, 2008.

[20] G. Dogan, P. Morin, R. H. Nochetto, and M. Verani. Discrete gradient flows for shape optimization and applications. Computer methods in applied mechanics and engineering, 196(37):3898-3914, 2007. 
[21] M. Elimelech and W. A. Phillip. The future of seawater desalination: energy, technology, and the environment. science, 333(6043):712-717, 2011.

[22] L. Formaggia, J.-F. Gerbeau, F. Nobile, and A. Quarteroni. Numerical Treatment of Defective Boundary Conditions for the Navier-Stokes Equations. SIAM Journal on Numerical Analysis, 40(1):376-401, 2002.

[23] P. J. Frey and P.-L. George. Mesh generation: application to finite elements. ISTE, 2007.

[24] C. Fritzmann, J. Löwenberg, T. Wintgens, and T. Melin. State-of-the-art of reverse osmosis desalination. Desalination, 216(1):1 - 76, 2007.

[25] V. Girault and P.-A. Raviart. Finite Element Methods for Navier-Stokes Equations, volume 5 of Springer series in computational mathematics. Springer Verlag, 1986.

[26] S. Gravelle, L. Joly, F. Detcheverry, C. Ybert, C. Cottin-Bizonne, and L. Bocquet. Optimizing water permeability through the hourglass shape of aquaporins. Proceedings of the National Academy of Sciences, 110(41):16367-16372, 2013.

[27] S. Gravelle, L. Joly, C. Ybert, and L. Bocquet. Large permeabilities of hourglass nanopores: From hydrodynamics to single file transport. The Journal of chemical physics, 141(18):18C526, 2014.

[28] F. Hecht. New development in freefem++. J. Numer. Math., 20(3-4):251-265, 2012.

[29] A. Henrot and M. Pierre. Variation et optimisation de formes, volume 48. SpringerVerlag Berlin Heidelberg, 2005.

[30] A. Henrot and Y. Privat. Une conduite cylindrique n'est pas optimale pour minimiser l'énergie dissipée par un fluide. C. R. Math. Acad. Sci. Paris, 346(19-20):1057-1061, 2008.

[31] A. Henrot and Y. Privat. What is the optimal shape of a pipe? Arch. Ration. Mech. Anal., 196(1):281-302, 2010.

[32] J. G. Heywood, R. Rannacher, and S. Turek. Artificial boundaries and flux and pressure conditions for the incompressible navier-stokes equations. International Journal for Numerical Methods in Fluids, 22(5):325-352, 1996.

[33] G. Hummer, J. C. Rasaiah, and J. P. Noworyta. Water conduction through the hydrophobic channel of a carbon nanotube. Nature, 2001.

[34] I. Jaber and M. Ahmed. Technical and economic evaluation of brackish groundwater desalination by reverse osmosis (ro) process. Desalination, 165:209 - 213, 2004. Desalination Strategies in South Mediterranean Countries.

[35] G. Karniadakis, A. Beskok, and N. Aluru. Simple Fluids in Nanochannels. Springer, 2005.

[36] R. D. Letterman, A. W. W. Association, et al. Water quality and treatment. McGrawHill, 1999.

[37] A. Manzoni, A. Quarteroni, and G. Rozza. Shape optimization for viscous flows by reduced basis methods and free-form deformation. International Journal for Numerical Methods in Fluids, 70(5):646-670, 2012. 
[38] B. Mohammadi and O. Pironneau. Shape optimization in fluid mechanics. Annu. Rev. Fluid Mech., 36:255-279, 2004.

[39] B. Mohammadi and O. Pironneau. Applied shape optimization for fluids. Oxford university press, 2010.

[40] P. Morin, R. H. Nochetto, M. S. Pauletti, and M. Verani. Adaptive finite element method for shape optimization. ESAIM: Control, Optimisation and Calculus of Variations, 18(4):1122-1149, 2012.

[41] H. G. Park and Y. Jung. Carbon nanofluidics of rapid water transport for energy applications. Chem. Soc. Rev., 43(565), 2014.

[42] A. Quarteroni and G. Rozza. Optimal control and shape optimization of aortocoronaric bypass anastomoses. Mathematical Models and Methods in Applied Sciences, 13(12):1801-1823, 2003.

[43] F. Salehi. Current and future applications for nanofiltration technology in the food processing. Food and Bioproducts Processing, 92(2):161 - 177, 2014. Advances in Bioseparations for Food and Bioprocessing.

[44] K. Takata, T. Matsuzaki, and Y. Tajika. Aquaporins: water channel proteins of the cell membrane. Progress in Histochemistry and Cytochemistry, 39(1):1 - 83, 2004.

[45] H. van der Horst, J. Timmer, T. Robbertsen, and J. Leenders. Use of nanofiltration for concentration and demineralization in the dairy industry: Model for mass transport. Journal of Membrane Science, 104(3):205 - 218, 1995.

[46] A. S. Verkman. Aquaporins: translating bench research to human disease. Journal of Experimental Biology, 212(11):1707-1715, 2009.

[47] F. Vince, F. Marechal, E. Aoustin, and P. Bréant. Multi-objective optimization of ro desalination plants. Desalination, 222(1):96 - 118, 2008.

[48] F. Zhu, E. Tajkhorshid, and K. Schulten. Theory and simulation of water permeation in aquaporin-1. Biophysical Journal, 86(1):50-57, 2004. 